\title{
Aspects of Moduli Stabilization in Type IIB String Theory
}

\author{
Shaaban Khalil, Ahmad Moursy, and Ali Nassar \\ Center for Fundamental Physics, Zewail City of Science and Technology, Giza 12588, Egypt \\ Correspondence should be addressed to Ahmad Moursy; aemoursy@gmail.com
}

Received 1 October 2015; Revised 28 December 2015; Accepted 30 December 2015

Academic Editor: Elias C. Vagenas

Copyright (C) 2016 Shaaban Khalil et al. This is an open access article distributed under the Creative Commons Attribution License, which permits unrestricted use, distribution, and reproduction in any medium, provided the original work is properly cited. The publication of this article was funded by SCOAP ${ }^{3}$.

We review moduli stabilization in type IIB string theory compactification with fluxes. We focus on KKLT and Large Volume Scenario (LVS). We show that the predicted soft SUSY breaking terms in KKLT model are not phenomenological viable. In LVS, the following result for scalar mass, gaugino mass, and trilinear term is obtained: $m_{0}=m_{1 / 2}=-A_{0}=m_{3 / 2}$, which may account for Higgs mass limit if $m_{3 / 2} \sim \mathscr{O}(1.5) \mathrm{TeV}$. However, in this case, the relic abundance of the lightest neutralino cannot be consistent with the measured limits. We also study the cosmological consequences of moduli stabilization in both models. In particular, the associated inflation models such as racetrack inflation and Kähler inflation are analyzed. Finally, the problem of moduli destabilization and the effect of string moduli backreaction on the inflation models are discussed.

\section{Introduction}

Ever since the invention of the Kaluza-Klein mechanism, it was realized that extradimensional models are plagued with massless scalar fields when compactified to $4 \mathrm{D}$. In the original Kaluza-Klein construction, the radius of the $x^{5}$ circle, $R\left(x^{\mu}\right)$ is not fixed by the dynamics and appears as a scalar field with no potential in the effective $4 \mathrm{D}$ theory. This is a generic feature of most compactifications of higherdimensional gravitational/Yang-Mills theories. The parameters which describe the shape and size of the compactification manifold give rise to massless scalar moduli with no potential at tree level in 4D (flat directions), that is, nonstabilized moduli fields. These moduli are gravitationally coupled and as such their existence would be in conflict with experiment (see [1] for a review).

String theory being a candidate for a unified theory of the forces of nature must be able to reproduce the physics of our real world which is 4-dimensional with a small positive cosmological constant and chiral gauge interactions. This amounts to finding de Sitter vacua of string theory with all moduli stabilized. The seminal work in [2] constructed the first models of Grand Unified Theories (GUTs) from Calabi-Yau compactification of the heterotic string. In these models, the 10D gauge group $\left(E_{8} \times E_{8}\right.$ or $\left.S O(32)\right)$ is broken to Standard-Model-like gauge groups by turning on background gauge fields on the internal space. The chiral fermions are obtained from the dimensional reduction of the 10D gaugino and the number of the generations is half the Euler characteristic of the internal space [2]. These compactifications are purely geometric and give rise to an $\mathcal{N}=1$ supersymmetric (SUSY) 4D theories with large number of moduli. Other compactifications which leads to $\mathcal{N}=1$ SUSY in $4 \mathrm{D}$ are type II theories on Calabi-Yau orientifolds with D-branes and fluxes, M-theory on manifolds with $G_{2}$ holonomy, and F-theory on Calabi-Yau fourfold (see [3] for a review and references therein).

One essential fact about our universe is that it has a small positive cosmological constant, that is, a de Sitter space-time. The moduli scalar potential $V(\phi)$ should be such that its minimum produces the observed value of the cosmological constant. This turns out to be a very difficult problem since de Sitter vacua are known to break supersymmetry. The first attempt at finding realistic vacua of string theory was mainly concerned with Minkowski and Anti-de Sitter vacua [2, 46]. It was shown that turning on magnetic fluxes in the internal manifold leads to a nontrivial warp factor and a nonKähler geometry. The situation improved drastically since 
the introduction of D-branes as nonperturbative objects in string theory. This resulted in the celebrated KKLT scenario in which a moduli fixing mechanism was introduced [7]. The Large Volume Scenario followed after [8]. Therein, one can turn on a vacuum expectation value for the fluxes in the internal space without breaking the $4 \mathrm{D}$ Lorentz invariance.

In this paper, we review the flux compactifications and moduli stabilization in type IIB string theory. The KachruKallosh-Linde-Trivedi (KKLT) [7] scenario was a major step in constructing $\mathrm{dS}$ vacua with all the moduli stabilized including the volume modulus. The Large Volume Scenario (LVS) by Quevedo et al. $[9,10]$ was proposed as an alternative to stabilize all the moduli with the volume moduli stabilized at extremely large volume. Realistic models of moduli stabilization must come as close as possible to the observed phenomenology at low energy and also to account for cosmological inflation at high energy scales. From this point of view, we analyze the low energy phenomenology of both KKLT and LVS. We emphasize that these models provide specific set of soft SUSY breaking terms, which are not phenomenologically viable. In addition, we study the impact of these scenarios on inflation. It turns out that inflation could destabilize the moduli again. This problem has been analyzed in details in $[11,12]$. On the other hand, moduli stabilization may have backreaction effects on the inflationary potential, which could change the inflationary parameters.

This paper is organized as follows. In Section 2, we review the flux compactification and moduli stabilization in type IIB string theory. Section 3 is devoted to KKLT model and its variants, where we discussed several examples for vacuum uplifting. In Section 4, we present the LVS as an alternative scenario that overcomes some of the KKLT drawbacks. The phenomenological implications and SUSY breaking soft terms of these two models are studied in Section 5. In Section 6, we highlight the cosmological implications of these two models. Finally, we state our conclusions in Section 7.

\section{Flux Compactifications and Moduli Stabilization in String Theory}

We start by reviewing the heterotic string compactification which has been considered to connect string theory to four-dimensional physics [2]. Then, we discuss type IIB flux compactifications, where the complex structure moduli (CSM) and the dilaton are stabilized by the RR and NS-NS 3-form fluxes.

2.1. Heterotic String Compactification. To compactify string theory down to $4 \mathrm{D}$, one looks for vacuum solutions of the form $M_{10}=M_{4} \times M_{6}$, where $M_{4}$ is assumed to have $4 \mathrm{D}$ Poincaré invariance and $M_{6}$ (or simply $\mathscr{M}$ ) is a compact internal 6D Euclidean space. The most general metric compatible with these requirements can be written as [2]

$$
G_{M N}=\left(\begin{array}{cc}
e^{A(y)} \eta_{\mu \nu} & 0 \\
0 & g_{m n}(y)
\end{array}\right),
$$

where $y^{m}$ are the coordinates on $\mathscr{M}$ with the metric $g_{m n}(y)$ and the requirement of Poincaré symmetry of $M_{4}$ still allows for a warp factor which depends on $\mathscr{M}$ only.

We also require an $\mathcal{N}=1$ supersymmetry in $4 \mathrm{D}$ since this is phenomenologically appealing and gives more analytic control. For $M_{4}$, one considers homogenous and isotropic maximally symmetric solutions which implies that the Riemann tensor takes the form

$$
R_{\mu \nu \rho \lambda}=c\left(g_{\mu \rho} g_{\nu \lambda}-g_{\mu \lambda} g_{\nu \rho}\right)
$$

where $c$ is fixed by contracting both sides with $g^{\mu \rho} g^{\nu \lambda}$ and turns out to be equal to $R / 12$. The constant scalar curvature $R$ could be $R=0$ (Minkowski), $R<0$ (AdS), or $R>0$ (dS).

When the radius of curvature of $\mathscr{M}$ is large compared to the Planck scale, one can use the supergravity approximation of string theory. In order to have a supersymmetric background, the supergravity transformations of the fermions must vanish [2]

$$
\delta_{\varepsilon}(\text { Fermions })=0 \text {. }
$$

For heterotic string theory, the supergravity variations of the fermions are given by [2]

$$
\begin{aligned}
\delta \psi_{M} & =\nabla_{M} \varepsilon-\frac{1}{4} \mathbf{H}_{M} \mathcal{\varepsilon}, \\
\delta \lambda & =-\frac{1}{2} \not \partial \Phi \varepsilon+\frac{1}{4} \mathbf{H} \varepsilon, \\
\delta \xi & =-\frac{1}{2} \mathbf{F} \mathcal{\varepsilon},
\end{aligned}
$$

where $\psi_{M}, \lambda$, and $\xi$ are the gravitino, dilatino, and the gaugino, respectively. The $H$-flux is $\mathbf{H}_{M}=\gamma_{N P} H_{M N P}$ and $\mathbf{H}=\gamma_{M N P} H_{M N P}$; and $\mathbf{F}=\gamma_{M N} F_{M N}$ is Yang-Mills field strength of the $E_{8} \times E_{8}$ or $S O(32)$ gauge fields in $10 \mathrm{D}$.

The Bianchi identity of $H$ is given by

$$
d H=\frac{\alpha^{\prime}}{4}[\operatorname{Tr}(R \wedge R)-\operatorname{Tr}(F \wedge F)] .
$$

Since $d H$ is exact, that is, zero in cohomology, then the cohomology classes of $\operatorname{Tr}(R \wedge R)$ and $\operatorname{Tr}(F \wedge F)$ are the same.

The vanishing of the above variations for a given spinor $\varepsilon$ will put some restrictions on the background fields and in particular on the geometry and topology of $\mathscr{M}$. The compactification of heterotic string theory with vanishing $H$ flux (or vanishing torsion) was first studied in $[2,5]$ and it leads to the following conditions on the string background:

$$
\begin{aligned}
\delta \psi_{\mu} & =0 \longrightarrow G_{\mu \nu}=\eta_{\mu \nu}, \\
e^{A(y)} & =\text { constant. }
\end{aligned}
$$

That is, the external space $M_{4}$ is Minkowski with a constant warp factor.

The dilatino variation gives

$$
\partial_{m} \Phi=0 .
$$

That is, the dilaton is constant over $\mathscr{M}$. 
The gravitino variation $\delta \psi_{m}$ gives

$$
\nabla_{m} \varepsilon=0 .
$$

This equation says that $\mathscr{M}$ admits a covariantly constant spinor. The integrability condition resulting from the above equation implies that $\mathscr{M}$ is Ricci flat

$$
R_{m n}=0 .
$$

Hence, the first Chern class of $\mathscr{M}$ vanishes

$$
c_{1}=\frac{1}{2 \pi}[\mathscr{R}] \text {. }
$$

It was conjectured by Calabi and proved by Yau that Ricciflat compact Kähler manifolds with $c_{1}=0$ admit a metric with SU (3) holonomy. These metrics come in families and are parameterized by continuous parameters $T_{i}$ which defines the shape and sizes of $\mathscr{M}$. The parameters $T_{i}$ appear as scalar fields (moduli) in $4 \mathrm{D}$ with no potential and a major goal in string theory is to generate a potential which stabilizes these moduli in a way that is consistent with observations.

One can describe the $4 \mathrm{D} \mathscr{N}=1$ models resulting from the heterotic string compactification in terms of an effective SUSY theory. This theory is characterized by a Kähler potential $K$, a gauge kinetic function $f$, and a superpotential $W$. The tree-level superpotential $W$ does not fix the moduli. Due to nonrenormalization theorems, $W$ is not renormalized at any order in perturbation theory $[14,15]$. This means that if supersymmetry is unbroken at tree level, it will remain unbroken at all orders of perturbation theory. Nonperturbative effects such as gaugino condensation [16] can correct the superpotential and fix some of the moduli.

It is worth mentioning that there is an alternative way to stabilize the moduli if the string model is nonsupersymmetric. In this case, perturbative corrections can generate a one-loop potential for the moduli, for example, a vacuum energy. For example, in the nonsupersymmetric tachyonfree heterotic string models studied in [17-19], the oneloop vacuum energy was shown to be finite and extremized at the symmetry-enhancement points in the moduli space. The one-loop potential can stabilize some of the moduli fields. Another way to generate masses for the moduli is by the breaking of supersymmetry through a Scherk-Schwartz mechanism [20].

2.2. Type IIB Compactification. We now turn our discussion to type IIB string theory. The massless bosonic spectrum of type IIB consists of the metric $g_{M N}$, RR 0 -form $C_{0}$, and scalar dilaton $\phi$ which are combined into the axiodilaton $S=$ $C_{0}+i e^{-\phi}$, where the string coupling is given by $1 / g_{s}=e^{-\phi}$. In addition, the spectrum contains RR 2 -form $C_{2}$ and 4 -form $C_{4}$, as well as the NS 2-form $B_{2}$. It is convenient to combine the RR and NS 3-forms $F_{3}=d C_{2}$ and $H_{3}=d B_{2}$ into $G_{3}=F_{3}-S_{3}$. The classical action of type IIB supergravity $S_{\text {IIB }}^{0}$ is divided into a bulk action $S_{b}$, the Chern-Simons action $S_{\mathrm{cs}}$, and contributions from the D-brane sources $S_{l}$ [21] (more precisely, $S_{l}$ represents the action of the localized sources for the case of a D $p$-brane wrapping a $(p-3)$-cycle $\Sigma)$

$$
S_{\mathrm{IIB}}^{0}=S_{b}+S_{\mathrm{cs}}+S_{l} \text {. }
$$

In the string frame, $S_{b}, S_{\mathrm{cs}}$, and $S_{l}$ are given by

$$
\begin{aligned}
S_{b} & =\frac{1}{(2 \pi)^{7} \alpha^{4}} \int d^{10} x \sqrt{-g}\left\{e^{-2 \phi}\left[\mathscr{R}+4(\nabla \phi)^{2}\right]\right. \\
& \left.-\frac{F_{1}^{2}}{2}-\frac{1}{2 \cdot 3 !} G_{3} \cdot \bar{G}_{3}-\frac{\widetilde{F}_{5}^{2}}{4 \cdot 5 !}\right\}, \\
S_{\mathrm{cs}} & =\frac{1}{4 i(2 \pi)^{7} \alpha^{\prime 4}} \int e^{\phi} C_{4} \wedge G_{3} \wedge \bar{G}_{3}, \\
S_{l} & =\sum_{\text {sources }}\left(-\int_{\mathbb{R}^{4} \times \Sigma} d^{p+1} \xi T_{p} e^{-\phi} \sqrt{-g}\right. \\
& \left.+\mu_{p} \int_{\mathbb{R}^{4} \times \Sigma} C_{p+1}\right),
\end{aligned}
$$

where $T_{p}$ and $\mu_{p}$ are, respectively, the tension and charge of the $\mathrm{D} p$-brane. The string tension is expressed in terms of string length as

$$
\alpha^{\prime}=\frac{1}{M_{\mathrm{st}}^{2}}=\left(\frac{l_{s}}{2 \pi}\right)^{2} .
$$

The 5 -form $\widetilde{F}_{5}$ is defined as

$$
\widetilde{F}_{5}=d C_{4}-\frac{1}{2} C_{2} \wedge H_{3}+\frac{1}{2} F_{3} \wedge B_{2}
$$

which is self dual and satisfies Bianchi identity

$$
d \widetilde{F}_{5}=H_{3} \wedge F_{3}
$$

One would like to consider warped compactifications of type IIB on a compact manifold $\mathscr{M}$. The metric ansatz for a $4 \mathrm{D}$ warped compactification is given by [22]

$$
\begin{aligned}
d s_{10}^{2} & =\sum_{M, N=0}^{9} G_{M N} d x^{M} d x^{N} \\
& =e^{2 A(y)} \eta_{\mu \nu} d x^{\mu} d x^{\nu}+e^{-2 A(y)} g_{m n} d y^{m} d y^{n} .
\end{aligned}
$$

The $10 \mathrm{D}$ Einstein equation of motion is

$$
R_{M N}=\kappa^{2}\left(T_{M N}-\frac{1}{8} G_{M N} T\right),
$$

where

$$
T_{M N}=T_{M N}^{\text {sugra }}+T_{M N}^{l}
$$

is the total stress tensor of supergravity plus the localized objects; that is

$$
T_{M N}^{l}=-\frac{2}{\sqrt{-G}} \frac{\delta S_{l}}{\delta G^{M N}} .
$$


In this regard, the space-time components of the latter action reduce to

$$
\begin{aligned}
\nabla^{2} e^{4 A}= & \frac{e^{2 A}}{12 \operatorname{Im} \tau}\left|G_{3}\right|^{2}+e^{-6 A}\left(|\partial \alpha|^{2}+\left|\partial e^{4 A}\right|^{2}\right) \\
& +\frac{\kappa_{10}^{2}}{2} e^{2 A}\left(T_{m}^{m}-T_{\mu}^{\mu}\right)^{l},
\end{aligned}
$$

where $\alpha$ is a function on the compact space. Integrating both sides of this equation over the compact manifold $\mathscr{M}$, the lefthand side gives zero since it is a total derivative. If there are no localized sources, then the right-hand side is a sum of positive terms and vanishes only if $\alpha$ and $A$ are constants and $G_{3}=0$. This is the familiar no-go theorem of flux compactifications $[23,24]$. However, the existence of localized sources in string theory like orientifold planes can balance the contribution coming from fluxes to give a nontrivial warp factor. This was realized in string theory in [22]. The setup in [22] allows for a stabilization of complex structure moduli by turning on RR and NS fluxes in the internal space $[25,26]$.

2.3. Type IIB Fluxes and Moduli Stabilization. One way to see the problem in a simple setting is nicely reviewed in [25, 27] in a toy model and we review it here. One can generate a potential for the moduli by turning on fluxes in the internal space. The potential in $4 \mathrm{D}$ results from the Maxwell term of the fluxes

$$
\begin{aligned}
V & =\int_{\mathscr{M}} \sqrt{-g} g^{\mu_{1} v_{1}} \cdots g^{\mu_{p} v_{p}} F_{\mu_{1} \cdots \mu_{p}} F_{v_{1} \cdots v_{p}} \\
& =\int F_{p} \wedge * F_{p}
\end{aligned}
$$

where $g$ is the metric of the internal space. The metric $g$ will depend on the moduli of the internal space and, after doing the integral on $\mathscr{M}$, one gets a potential for the moduli $V(\phi)$. For example, consider a 6D Maxwell-Einstein theory compactified on a two-sphere $S^{2}$ with a nonzero flux of $F_{2}$ piercing $S^{2}$

$$
\int_{S^{2}} F=N
$$

This flux contributes a positive energy to the effective 4D potential which can then balance the negative contribution coming from the curvature of $S^{2}$. More specifically, the contribution to the effective potential coming from the flux originates from the Maxwell term in 6D

$$
V(R)=\int F_{2} \wedge * F_{2} \sim \frac{N^{2}}{R^{6}},
$$

where the determinant of the metric contributes a factor of $R^{2}$ and two metric contractions contribute a factor of $1 / R^{2}$ while the transformation to the Einstein frame gives a factor $1 / R^{4}$. Therefore, total $4 \mathrm{D}$ potential takes the form

$$
V(R)=\frac{N^{2}}{R^{6}}-\frac{1}{R^{4}},
$$

which is minimized at $R=N$, and if $N$ is large the curvature is small and the supergravity approximation is reliable $[25,27]$. In string theory, additional ingredients beside the fluxes are needed to construct stable vacua. These ingredients are the D-branes and orientifold planes [28].

The main idea of flux compactification is that there are solutions of the string tree-level equations in which some of the $p$-form fields are nonzero in the vacuum. In these constructions, one needs to make sure that the backreaction of the flux on the geometry does not take us outside the supergravity approximation. This turns out to be possible [5] with the introduction of a warp factor varying over the internal manifold and hence the new geometry is conformal to the nonflux case. The fluxes, which can be turned on, are the RR fluxes of type II and the $H_{3}$ flux. In this case, the quantization condition on the fluxes is

$$
\frac{1}{l_{s}^{p}} \int_{\Sigma_{p+1}} F_{p+1} \in \mathbb{Z},
$$

where the integrality of the cohomology classes of $F_{p+1}$ is due to Dirac's charge quantization. The nonvanishing of the cohomology classes of these $p$-form fields leads to obstructions which lifts some of the flat directions of the compactification; that is, it leads to potential which freezes some of the moduli.

In the presence of sources, the modified Bianchi identity now reads [28]

$$
d \widetilde{F}_{5}=H_{3} \wedge F_{3}+2 \kappa_{10}^{2} \mu_{3} \sum_{a} \pi_{6}^{a}+2 \kappa_{10}^{2} Q_{3} \mu_{3} \pi_{6}^{\mathrm{O} 3} .
$$

Integrating this equation over the compact internal manifold, one gets the tadpole cancelation condition [28]

$$
N_{\text {flux }}+N_{\mathrm{D} 3}+Q_{3} N_{\mathrm{O} 3}=0
$$

where

$$
N_{\text {flux }}=\frac{1}{l_{s}^{4}} \int_{\mathscr{M}} H_{3} \wedge F_{3} .
$$

The type IIB string theory will be compactified on Calabi-Yau orientifolds in order to obtain a $4 \mathrm{D} \mathcal{N}=1$ model. It turned out that one needs to make an orientifold projection in order to have supersymmetric compactification [28]. The orientifold action projects out one of the two gravitinos and breaks the $\mathcal{N}=2$ SUSY down to $\mathcal{N}=$ 1. The orientifold projection also introduces $\mathrm{O}$-planes with a background charge and a negative energy density which balances the contribution of D-branes and leads to stable compactification. The RR and NS-NS 3-form fluxes are restricted via the integral cohomology which determines the quantized background fluxes as follows:

$$
\begin{aligned}
& \frac{1}{(2 \pi)^{2} \alpha^{\prime}} \int_{\Sigma_{a}} F_{3}=n_{a} \in \mathbb{Z}, \\
& \frac{1}{(2 \pi)^{2} \alpha^{\prime}} \int_{\Sigma_{b}} H_{3}=m_{b} \in \mathbb{Z},
\end{aligned}
$$


where $\Sigma_{a, b}$ are 3 cycles of the Calabi-Yau manifold. In this case, tadpole condition (27) reads

$$
\frac{\chi(\mathscr{M})}{24}=N_{\mathrm{D} 3}+\frac{1}{\left(2 \pi^{4}\right) \alpha^{\prime 2}} \int_{\mathscr{M}} H_{3} \wedge F_{3},
$$

where $N_{\mathrm{D} 3}$ is the net number of (D3 $\left.-\overline{\mathrm{D} 3}\right)$ branes filling the noncompact dimensions and $\chi(\mathscr{M})$ is the Euler characteristic of the elliptically fibred Calabi-Yau fourfold $\mathscr{M}$.

The compactification to $4 \mathrm{D}$ on a Calabi-Yau manifold with orientifold planes will give rise to an $\mathcal{N}=1$ supergravity theory which is characterized by a Kähler potential $K$, a superpotential $W$, and a gauge kinetic function $f$ [2931]. The tree-level Kähler potential is given by the WeilPetersson metric using the Kaluza-Klein reduction of type IIB supergravity

$$
\begin{aligned}
K= & -3 \ln [(T+\bar{T})]-\ln [(S+\bar{S})] \\
& -\ln \left[-i \int_{\mathscr{M}} \Omega \wedge \bar{\Omega}\right] .
\end{aligned}
$$

Here, $T$ represents the volume modulus and is one of the Kähler moduli. The conditions on the fluxes in type IIB are

$$
\begin{aligned}
& * G_{3}=i G_{3}, \\
& G_{3}^{0,3}=0 .
\end{aligned}
$$

Since the hodge $*$ depends on the metric, then one expects the above conditions can fix the geometric moduli except for the overall scale of the metric since the hodge $*$ is conformally invariant in six dimensions. This leaves the overall volume of the compactification manifold undetermined. These conditions can be derived from a superpotential given by the Gukov-Vafa-Witten (GVW) form [32]

$$
W=\int_{\mathscr{M}} \Omega \wedge G_{3} .
$$

This superpotential depends on the complex structure moduli through $\Omega$ and is independent of the Kähler moduli.

The $N=1$ supergravity scalar potential is given by

$$
V=e^{K}\left(\sum_{a, b} g^{a \bar{b}} D_{a} W \overline{D_{b} W}-3|W|^{2}\right),
$$

where $a, b$ run over all the moduli. Due to the no-scale structure of the Kähler potential (31), the sum over Kähler moduli cancels the term $3|W|^{2}$ and the potential (34) reduces to the no-scale structure [33-35]

$$
V_{\text {no-scale }}=e^{K}\left(\sum_{i, j} g^{i \bar{j}} D_{i} W \overline{D_{j} W}\right) \text {, }
$$

where $i, j$ run over the dilaton and complex structure moduli. Accordingly, the dilaton and complex structure moduli can be stabilized in a supersymmetric minimum by solving the equation $D_{a} W=0$ which may have a solution for generic choice of the flux. In this case, $W=W_{0}=0$ at the minimum.
The above discussion shows that the no-scale structure does not fix the value of the volume modulus $T$; that is, the modulus $T$ is a flat direction. The stabilization of $T$ is of uttermost importance in order for string theory to make contact with realistic models. This issue will be addressed in the upcoming sections.

\section{KKLT and Its Variants}

In order to stabilize the volume modulus $T$, a nonperturbative superpotential was considered by Kachru, Kallosh, Linde, and Trivedi (KKLT) [7]. The source of these nonperturbative terms could be either D3-brane instantons or gaugino condensation from the nonabelian gauge sector on the D7branes. As advocated in the previous section, the dilaton and the complex structure moduli are stabilized at a high scale by the flux induced superpotential and hence their contribution to the superpotential is a constant $W_{0}$. Thus, the total effective superpotential is given by

$$
W=W_{0}+A e^{-a T} .
$$

The coefficient $a=2 \pi$ or $2 \pi / N$ is the correction arising from D3 instantons or $S U(N)$ gaugino condensation, and $A$ is constant of order $\mathcal{O}(1)$. In addition, the Kähler potential is given by

$$
K=-3 \ln [T+\bar{T}]
$$

Here, $T=\tau+i \psi$, where $\tau$ is the volume modulus of the internal manifold and $\psi$ is the axionic part. A supersymmetric minimum is obtained by solving the equation

$$
\begin{aligned}
D_{T} W & =0 \Longrightarrow \\
W_{0} & =-A e^{-a \tau_{0}}\left(1+\frac{2}{3} a \tau_{0}\right),
\end{aligned}
$$

where $\tau_{0}$ is the value of $\tau$ that minimizes the scalar potential.

Substituting this solution in the potential

$$
V=e^{K}\left(\frac{3}{(T+\bar{T})^{2}}\left|D_{T} W\right|^{2}-3|W|^{2}\right),
$$

one finds the following negative minimum:

$$
V_{0}^{\mathrm{AdS}}=\left.\left(-3 e^{K}|W|^{2}\right)\right|_{\tau_{0}}=-\frac{a^{2} A^{2} e^{-2 a \tau_{0}}}{6 \tau_{0}} .
$$

The scalar potential as a function of $\tau=\operatorname{Re}(T)$ is given by (the imaginary part $\operatorname{Im}(T)=\psi$ is frozen at zero)

$$
V(\tau)=\frac{a A e^{-2 a \tau}\left(A(a \tau+3)+3 W_{0} e^{a \tau}\right)}{6 \tau^{2}} .
$$

It is important to uplift this AdS minimum to a Minkowski or a dS minimum, as shown in Figure 1, in order to have realistic models. The uplift of the above AdS vacuum to a dS one will break SUSY where one needs another 


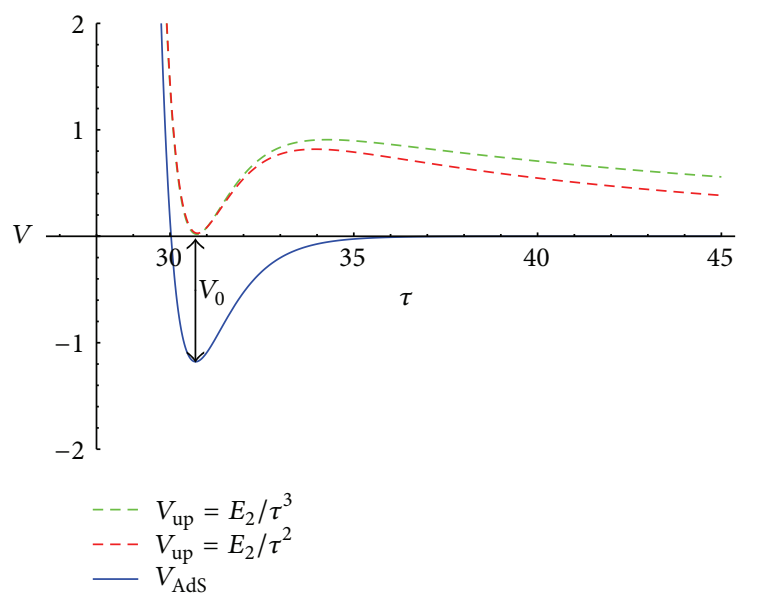

FIgURE 1: The scalar potential $V(\tau)$ (multiplied by $10^{29}$ ) with $W_{0}=$ $-10^{-12}, A=1$, and $a=1$. The blue curve shows the AdS minimum, while the green and red curves exhibit the uplifting to dS minimum via $\delta V=E_{3} / \tau^{3}, E_{2} / \tau^{2}$, respectively, with $E_{3}=3.5 \times 10^{-25}$ and $E_{2}=$ $1.13 \times 10^{-26}$.

contribution to the potential which usually has dependence like $\tau^{-n}[36]$

$$
\delta V(\tau) \approx\left|V_{0}^{\mathrm{AdS}}\right| \frac{\tau_{0}^{n}}{\tau^{n}}
$$

In this case, a new minimum is obtained due to shifting $\tau_{0}$ to $\tau_{0}^{\prime}=\tau_{0}+\varepsilon$, where $\varepsilon$ is given by

$$
\varepsilon \simeq \frac{1}{a^{2} \tau_{0}}
$$

Since the consistency of the KKLT requires that $\tau_{0}, a \tau_{0} \gg 1$ [7], the shift in the minima is much small and we can calculate physical quantities such as masses in terms of $\tau_{0}$.

There are many proposals for such uplifting, for example, adding anti-D3-branes [7], D-term uplift [37-43], F-term uplift [13, 44], and Kähler uplift [41, 45-47]. In the original KKLT scenario [7], some anti-D3-branes were added which contributes along with additional part to the scalar potential

$$
\delta V=\frac{E_{3}}{\tau^{3}}
$$

One of the drawbacks of this mechanism is that SUSY is broken explicitly due to the addition of the anti-D3-branes. In this case, the effective $4 \mathrm{D}$ theory cannot be recast into the standard form of $4 \mathrm{D}$ supergravity and this in turn makes it very difficult to have a low energy effective theory [37]. An uplifting mechanism via a D-term scalar potential was proposed in [37] where the possible fluxes of gauge fields living on the D7-branes were used. In this case, the fluxes induce a term in the $4 \mathrm{D}$ effective action of the form

$$
T_{7} \int_{\Sigma} d^{4} y \sqrt{g_{8}} F^{m n} F_{m n}=\frac{2 \pi E^{2}}{\tau^{3}}
$$

where $\Sigma$ is the 4 cycles on which D7-branes wrap, $T_{7}$ is the tension of D7-branes, and $E$ measures the strength of the flux. Accordingly, the D-term scalar potential is given by

$$
V_{D}=\frac{g_{Y M}^{2}}{2} D^{2}=\frac{2 \pi}{\tau}\left(\frac{E}{\tau}+\sum q_{i}\left|C_{i}\right|^{2}\right)^{2},
$$

where $C_{i}$ are charged matter fields with charges $q_{i}$. These fields can be minimized at $C_{i}=0$ and accordingly the full potential will be

$$
V=V_{F}+V_{D}
$$

with $V_{D}=2 \pi E^{2} / \tau^{3}$ and we end up with similar behavior to the KKLT uplifted potential.

Another approach for uplifting uses the F-term uplifting $[13,44]$. In this case, SUSY will be broken spontaneously in the F-term moduli sector which in turn generates an uplift term for the AdS KKLT stabilized volume. For example, in [13], the Kähler potential contains a modulus $T$ and a matter field $C$ and has the form

$$
K=-3 \ln (T+\bar{T})+|C|^{2} .
$$

The effective superpotential [13] is given by

$$
W=\sum_{i} \omega_{i}(C) e^{-\alpha_{i} T}+\phi(C),
$$

where $\omega_{i}(C)$ and $\phi(C)$ are functions of the matter fields resulting due to integrating out heavy fields with index $i$ that runs over the gaugino condensates. The scalar potential will be minimized at [13]

$$
\begin{aligned}
& C=0, \\
& T=T_{0} .
\end{aligned}
$$

Figure 2 shows the shape of the potential near the minimum $T=T_{0}, C=0$. This model is different from the approach studied in [48], where the effects of charged chiral fields that reside on D3-branes and D7-branes [49] were considered. In that case, new $T$-dependence will be generated in the Kähler potential

$$
\begin{aligned}
K= & -3 \ln (T+\bar{T})-3 \ln (S+\bar{S})+\frac{\left|\left\langle C_{3}\right\rangle\right|^{2}}{(T+\bar{T})} \\
& +\frac{\left|\left\langle C_{7}\right\rangle\right|^{2}}{(S+\bar{S})},
\end{aligned}
$$

where $C_{3}, C_{7}$ are charged chiral matter fields. In addition, the superpotential does not contain any nonperturbative effects

$$
W=A+B S .
$$

Therefore, an AdS minimum is obtained which is uplifted by a D-term associated with the gauge symmetry of the matter fields.

Another way for uplifting to dS vacua of the volume stabilized moduli is by Kähler uplift models where the perturbative 


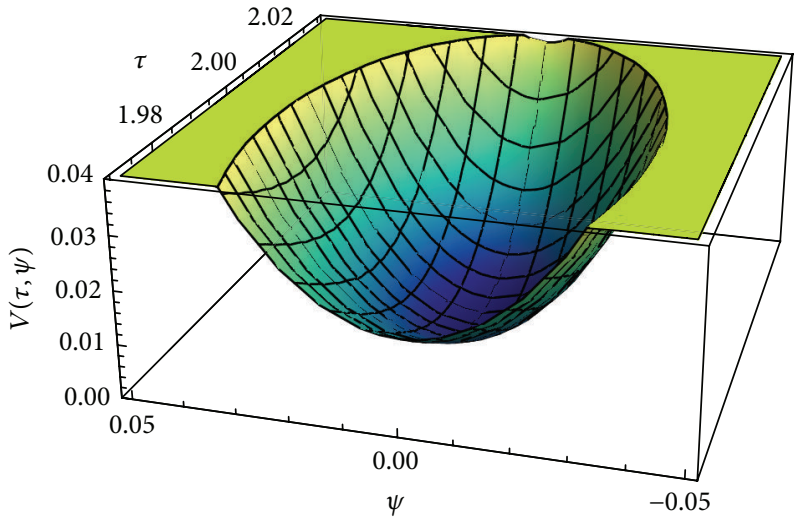

(a)

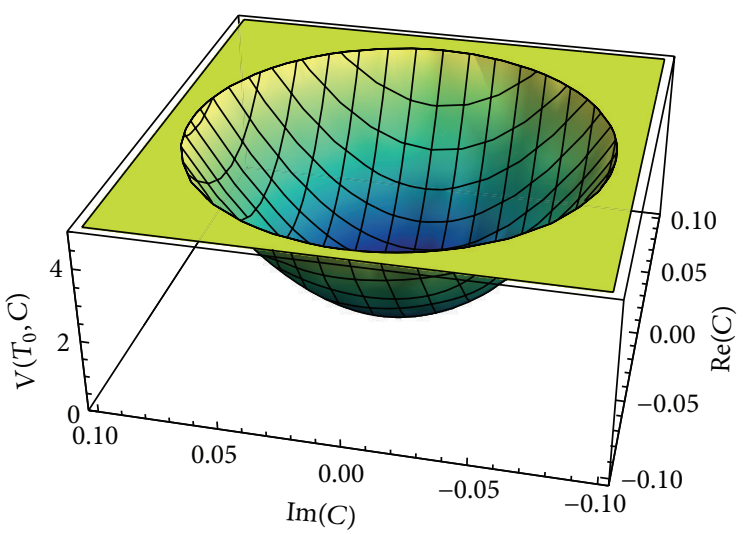

(b)

Figure 2: The minimum of the potential of the scenario [13] at $T=2, C=0$. (a) corresponds to $V(T, 0)$ rescaled by $1 / \epsilon^{2}$ and (b) corresponds to $V\left(T_{0}, C\right)$ rescaled by $10^{4} / \epsilon^{2}$.

corrections to the Kähler potential will paly an important role in constructing dS vacua [41, 45-47]. Now, we consider a model proposed in [46] where $h^{1,1}=1$ and $h^{2,1}>1$ so that the Euler characteristic is $\chi=2\left(h^{1,1}-h^{2,1}\right)<0$. In this case, the Kähler potential and superpotential are

$$
\begin{aligned}
& K=-2 \ln \left(\widehat{\mathscr{V}}+\alpha^{\prime 3} \frac{\widehat{\xi}}{2}\right), \\
& W=W_{0}+\sum_{i} A_{i} e^{-a_{i} T_{i}},
\end{aligned}
$$

where $\widehat{\mathscr{V}}=\gamma(T+\bar{T})^{3 / 2}$ is the normalized volume, with $\gamma=$ $\sqrt{3} /(2 \sqrt{\kappa})$ and

$$
\widehat{\xi}=-\frac{\chi(\mathscr{M}) \zeta(3)}{4 \sqrt{2}(2 \pi)^{3}}(S+\bar{S})^{3 / 2}
$$

Accordingly, the $\tau$ dependence of $N=1$ supergravity scalar potential is given by $[46,50,51]$

$$
\begin{aligned}
& V(t) \\
& \quad=e^{K}\left(K^{T \bar{T}}\left[a^{2} A^{2} e^{-2 a t}+\left(-a A e^{-a t} \overline{W K_{T}}+\text { c.c }\right)\right]\right. \\
& \left.+3 \widehat{\xi} \frac{\widehat{\xi}^{2}+7 \widehat{\xi} \widehat{\mathscr{V}}+\widehat{\mathscr{V}}^{2}}{(\widehat{\mathscr{V}}-\widehat{\xi})(\widehat{\xi}+2 \widehat{\mathscr{V}})^{2}}|W|^{2}\right),
\end{aligned}
$$

where $\psi$ stabilizes at $\psi=n \pi / a$ for $n=0,1, \ldots$ Using the approximations $\widehat{\mathscr{V}} \gg \widehat{\xi}$ and $\left|W_{0}\right| \gg A e^{-a t}$ and defining the quantities

$$
\begin{aligned}
& x=a \cdot t, \\
& C=\frac{-27 W_{0} \widehat{\xi} a^{3 / 2}}{64 \sqrt{2} \gamma A},
\end{aligned}
$$

the scalar potential will be simplified to the form

$$
V(x) \simeq \frac{-W_{0} a^{3} A}{2 \gamma^{2}}\left(\frac{2 C}{9 x^{9 / 2}}-\frac{e^{-x}}{x^{2}}\right) .
$$

In order to have stable $\mathrm{dS}$ vacuum, $C$ must satisfy the constraint [46]

$$
3.65 \lesssim C \lesssim 3.89 .
$$

This is clarified in Figure 3, where, for values of $C<3.65$, we have AdS minimum and, for values of $C>3.89$, the volume is destabilized.

\section{Moduli Stabilization in Large Volume Scenario}

Another alternative scenario for moduli stabilization based on a Large Volume Scenario has been proposed by Quevedo et al. $[9,10]$ in order to overcome some of the drawbacks of the KKLT model. Basically, increasing the number of Kähler moduli will worsen the situation when $\alpha^{\prime}$ corrections are neglected. The LVS was built on the proposal that the number of complex structure moduli is bigger than the number of Kähler moduli; that is, $h^{2,1}>h^{1,1}>1$, as well as the inclusion of $\alpha^{\prime}$ corrections. $\mathcal{O}\left(\alpha^{\prime 3}\right)$ contribution to the Kähler potential (after integrating out the dilaton and the complex structure moduli) is given by [50-52]

$$
K_{\alpha^{\prime}}=-2 \log \left[e^{-3 \phi / 2} \mathscr{V}+\frac{\xi}{2}\left(\frac{(S+\bar{S})}{2}\right)^{3 / 2}\right]+K_{\mathrm{cs}},
$$

with $\xi=-\zeta(3) \chi(\mathscr{M}) / 2(2 \pi)^{3}$ and $\phi$ is the type IIB dilaton. Here, $\mathscr{V}$ is defined as the classical volume of the manifold $\mathscr{M}$ which is given by

$$
\mathscr{V}=\int_{\mathscr{M}} J^{3}=\frac{1}{6} \kappa_{i j k} t^{i} t^{j} t^{k}
$$




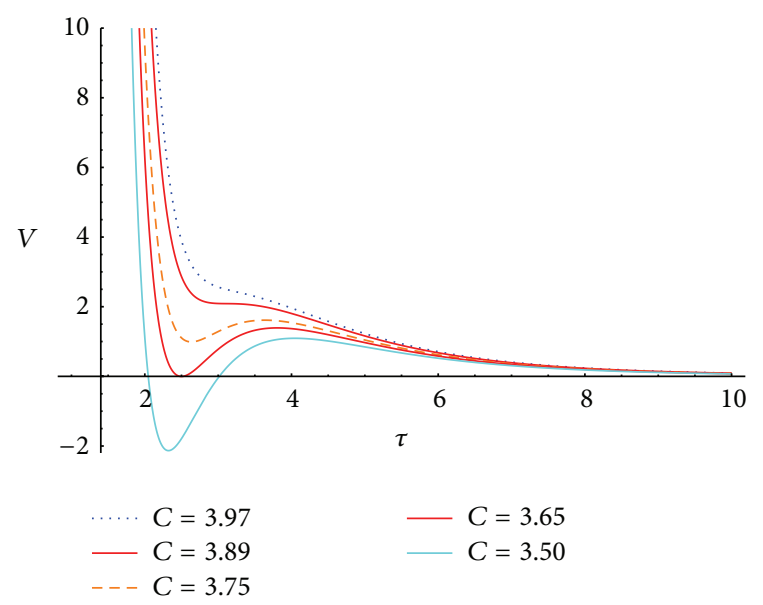

Figure 3: Potential of one modulus model for the Kähler uplift. The red curves correspond to the limits on the parameter $C$ for a dS vacuum, while the dotted one corresponds to $C$ in the destabilization region.

where $J$ is the Kähler class and $t_{i}$ are the moduli that measure the areas of 2 cycles with $i=1,2, \ldots, h_{1,1}$. The complexified Kähler moduli are defined as $T_{j} \equiv \tau_{j}+i \psi_{j}$, where $\tau_{j}$ are the four-cycle moduli defined by the relation

$$
\tau_{j}=\partial_{t_{j}} \mathscr{V}=\frac{1}{2} \kappa_{j k l} t^{k} t^{l}
$$

Since the superpotential is not renormalized at any order in perturbation theory, it will not receive $\alpha^{\prime}$ corrections. But there is a possibility of nonperturbative corrections, which may depend on the Kähler moduli (as in the KKLT model) via D3-brane instantons or gaugino condensation from wrapped D7-branes. Accordingly, the superpotential is given by

$$
W=W_{0}+\sum_{i} A_{i} e^{-a_{i} T_{i}}
$$

where $A_{i}$ are model dependent constants and again $W_{0}$ is the value of the superpotential due to the geometric flux after stabilizing the dilaton and the complex structure moduli. In this respect, the scalar potential will take the form $[50,51]$

$$
\begin{aligned}
V= & e^{K}\left[K ^ { T _ { j } \overline { T } _ { k } } \left(a_{j} A_{j} a_{k} \bar{A}_{k} e^{\left(a_{j} T_{j}+a_{k} \bar{T}_{k}\right)}\right.\right. \\
& \left.+a_{j} A_{j} e^{i a_{j} T_{j}} \bar{W} \partial_{\bar{T}_{k}} K-a_{k} \bar{A}_{k} e^{-i a_{k} \bar{T}_{k}} W \partial_{T_{j}} K\right)+3 \xi \\
& \left.\cdot \frac{\left(\xi^{2}+7 \xi \mathscr{V}+\mathscr{V}^{2}\right)}{(\mathscr{V}-\xi)(2 \mathscr{V}+\xi)^{2}}|W|^{2}\right] \equiv V_{n p 1}+V_{n p 2}+V_{\alpha^{\prime}}
\end{aligned}
$$

where $\alpha^{\prime}$ correction is encoded in $V_{\alpha^{\prime}}$. The simplest example that can realize the notion of LVS $[9,10]$ is the orientifold of $P_{[1,1,1,6,9]}^{4}$ for which $h^{1,1}=2$ and $h^{2,1}=272$ and therefore the volume is given by

$$
\mathscr{V}=\frac{1}{9 \sqrt{2}}\left(\tau_{5}^{3 / 2}-\tau_{4}^{3 / 2}\right)
$$

where the volume moduli are $T_{4}=\tau_{4}+i \psi_{4}$ and $T_{4}=\tau_{5}+i \psi_{5}$ and the link to $t_{i}$ is given by $\tau_{4}=t_{1}^{2} / 2$ and $\tau_{5}=\left(t_{1}+6 t_{5}\right)^{2} / 2$. In this respect, the Kähler potential and the superpotential, after fixing the dilaton and complex structure moduli, are given in the string frame by

$$
\begin{aligned}
& K=K_{\mathrm{cs}}-2 \log \left(\mathscr{V}+\frac{\xi}{2}\right) \\
& W=W_{0}+A_{4} e^{-\left(a_{4} / g_{s}\right) T_{4}}+A_{5} e^{-\left(a_{5} / g_{s}\right) T_{5}} .
\end{aligned}
$$

In the large volume limit, $\mathscr{V} \sim \tau_{5} \gg \tau_{4}>1$, the behavior of the scalar potential is given by $[9,10]$

$$
\begin{aligned}
V\left(\mathscr{V}, \tau_{4}\right)= & \frac{\sqrt{\tau_{4}}\left(a_{4} A_{4}\right)^{2} e^{-2 a_{4} \tau_{4} / g_{s}}}{\mathscr{V}} \\
& -\frac{W_{0} \tau_{4} a_{4} A_{4} e^{-a_{4} \tau_{4} / g_{s}}}{\mathscr{V}^{2}}+\frac{\xi W_{0}^{2}}{\mathscr{V}^{3}} .
\end{aligned}
$$

Minimizing the potential (66), one finds the following:

$$
\frac{\partial V}{\partial \mathscr{V}}=\frac{\partial V}{\partial \tau_{4}}=0
$$

and solving the two equations in the two variables, $\tau_{4}, \mathscr{V}$, one can get one equation in $\tau_{4}$

$$
\left(1 \pm \sqrt{1-\frac{3 B_{3} B_{1}}{B_{2} \tau_{4}^{3 / 2}}}\right)\left(\frac{1}{2}-2 a_{4} \tau_{4}\right)=\left(1-a_{4} \tau_{4}\right)
$$

where

$$
\begin{aligned}
& B_{1} \sim a_{4}^{2}\left|A_{4}\right|^{2}, \\
& B_{2} \sim a_{4}\left|A_{4} W_{0}\right|, \\
& B_{3} \sim \xi\left|W_{0}\right|^{2} .
\end{aligned}
$$

Using the assumption $a_{4} \tau_{4} \gg 1$, which is necessary to neglect higher order instanton corrections [9], the solution is given by

$$
\begin{aligned}
& \tau_{4}=\left(\frac{4 B_{3} B_{1}}{B_{2}^{2}}\right)^{2 / 3}, \\
& \mathscr{V}=\frac{B_{2}}{2 B_{1}}\left(\frac{4 B_{3} B_{1}}{B_{2}^{2}}\right)^{1 / 3} e^{a_{4}\left(4 B_{3} B_{1} / B_{2}^{2}\right)^{2 / 3}} .
\end{aligned}
$$

Substituting for $B_{i}$ by their expressions, we have

$$
\begin{aligned}
& \tau_{4} \sim(4 \xi)^{2 / 3}, \\
& \mathscr{V} \sim \frac{\xi^{1 / 3}\left|W_{0}\right|}{a_{4} A_{4}} e^{a_{4} \tau_{4} / g_{s}} .
\end{aligned}
$$

Therefore, the potential possesses an AdS minimum at exponentially large volume $\mathscr{V}$ since it approaches zero from 


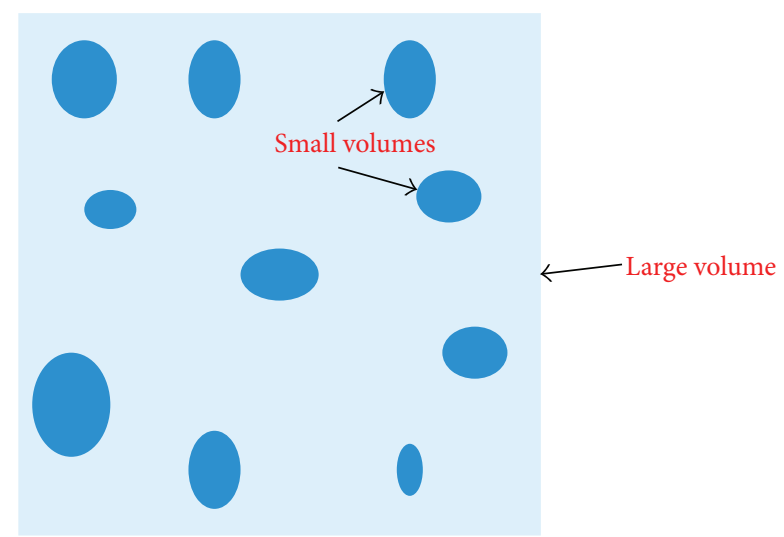

FIgURE 4: Swiss-cheese structure in Large Volume Scenario.

below in the limit $\tau_{5} \rightarrow \infty$ and $\tau_{4} \propto \log (\mathscr{V})$. Namely, in the latter limit, the potential has the form

$$
V=\frac{W_{0}^{2}}{\mathscr{V}^{3}}\left(C_{1} \sqrt{\ln (\mathscr{V})}-C_{2} \ln (\mathscr{V})+\xi \mathscr{V}\right) .
$$

Therefore, the negativity of the potential requires a very large $\ln (\mathscr{V})$. Still one has to uplift this minimum by one of the mechanisms mentioned in Section 3. This result can be generalized to more than two moduli where one of them takes a large limit while other moduli stay small. This structure will form what is called the Swiss-cheese form of the CY manifold, as depicted in Figure 4. In this case, the volume will take the form

$$
\mathscr{V}=\tau_{b}^{3 / 2}-\sum_{i} \tau_{s, i}^{3 / 2}
$$

\section{SUSY Breaking and \\ Phenomenological Consequences}

In this section, we will study SUSY breaking in the moduli sector and the properties of the corresponding soft terms (more general expressions of soft term for generic superpotentials and Kähler potentials in supergravity and string models are given in $[53,54]$.) that are induced in the observable sector, and then we summarize the phenomenological consequences. SUSY breaking in models of KKLT compactification type with phenomenological consequences has been extensively studied in [36, 40, 55-59], while, for LVS type, SUSY breaking was studied in [10, 60-65]. We would like to remark that in studying MSSM-like theories arising from large volume compactifications one faces the issue of the validity of perturbation theory. In large volume models, one has to worry about the Kaluza-Klein towers which become light when the compactification volume is large. If some of these KK modes are charged under the gauge symmetry, they can push the gauge and Yukawa couplings to large values and the perturbative control is lost.

(i) Soft Terms in KKLT Scenario. As shown in models of KKLT type in Section 3, SUSY is broken by one of the uplifting mechanisms. The gravitino mass at the $\mathrm{dS}$ minimum is given by

$$
\begin{aligned}
& m_{3 / 2}=\left.e^{K / 2}|W|\right|_{\mathrm{dS}} \Longrightarrow \\
& m_{3 / 2} \simeq \frac{a A}{3\left(2 \tau_{0}\right)^{1 / 2}} e^{-a \tau_{0}} \simeq \frac{W_{0}}{\left(2 \tau_{0}\right)^{3 / 2}}
\end{aligned}
$$

Therefore, we have gravitino mass of order $\sim 1 \mathrm{TeV}$ if $\left(a \tau_{0}\right) \sim$ 32 and hence $W_{0} \sim 10^{-12}$. In terms of the shifts $\varepsilon$ (given in (43)) of $\tau_{0}$, an approximate expression for $D_{T} W$ near the $\mathrm{dS}$ minimum is given by

$$
D_{T} W\left(\tau_{0}+\varepsilon\right)=\left(D_{T} W\right)_{\tau} \varepsilon \simeq W_{T, T} \varepsilon=\frac{3 \sqrt{2}}{a \sqrt{\tau_{0}}} m_{3 / 2}
$$

which is of the same order as the gravitino mass. Accordingly, the soft SUSY breaking terms are given by

$$
\begin{aligned}
m_{0}^{2} & =\left.\frac{|W|^{2}}{(2 \tau)^{3}}\right|_{\mathrm{dS}}=m_{3 / 2}^{2}, \\
m_{1 / 2} & =\left.\frac{\sqrt{2 \tau}}{6} D_{T} W(T) \frac{\partial}{\partial T} \ln \left(\operatorname{Re} f^{*}\right)\right|_{\mathrm{dS}} \simeq \frac{m_{3 / 2}}{a \tau}, \\
A_{0} & =-\left.\frac{1}{\sqrt{2 \tau}} \bar{D}_{\bar{T}} \overline{W_{h}}\right|_{\mathrm{dS}}=-\frac{3 m_{3 / 2}}{a \tau},
\end{aligned}
$$

where the gauge kinetic function $f_{a b}$ can be chosen such that $f_{a b}(T)=f(T) \delta_{a b}$, which will lead to universal gaugino masses. The gauge kinetic function is considered to have a linear dependance on the modulus field which can be derived from the reduction of Dirac-Born-Infeld action for an unmagnetized brane $[62,63]$.

The soft terms indicate that SUSY breaking in KKLT is a special example of the constrained MSSM (CMSSM), where all the soft terms are given in terms of one free parameter $\left(m_{3 / 2}\right)$ which is of order TeV. Note that $a \tau_{0}$ is fixed as $a \tau_{0} \simeq 32$. It is well known that this type of soft terms cannot account for the experimental constraints imposed by the Large Hadron Collider (LHC) and relic abundance of the lightest SUSY particle. Even if we relax the Dark matter constraints, the Higgs mass limit $(\sim 125 \mathrm{GeV})$ and gluino mass limit $(\gtrsim 1.4 \mathrm{TeV})$ will imply $m_{3 / 2} \simeq \mathcal{O}(30) \mathrm{TeV}$. Thus, all SUSY spectrum will be quite heavy which is beyond the LHC sensitivity. A feature of this model is the fact that gauginos are lighter than the sfermions by at least one order of magnitude. However, if one checks the parameter space for such set of soft terms, he finds that tadpole equations at the $\mathrm{TeV}$ scale are not satisfied. Namely, condition [66]

$$
\mu^{2}+\frac{M_{Z}^{2}}{2} \simeq-0.1 m_{0}^{2}+2 m_{1 / 2}^{2}
$$


cannot account for positive $\mu^{2}$. Therefore, the above set of soft terms failed to describe $\mathrm{TeV}$ scale phenomenology.

(ii) Soft Terms in LVS Scenario. Let us now consider the same scenario for SUSY breaking in LVS model, namely, with $P_{[1,1,1,6,9]}^{4}$ geometry. In this case, we have

$$
\begin{aligned}
K & =-2 \log \left(\mathscr{V}+\frac{\xi}{2}\right), \\
W & =W_{0}+A_{4} e^{-\left(a_{4} / g_{s}\right) T_{4}}+A_{5} e^{-\left(a_{5} / g_{s}\right) T_{5}} .
\end{aligned}
$$

Therefore, the gravitino mass will be given by

$$
m_{3 / 2}=\left.e^{K / 2}|W|\right|_{\mathrm{dS}} \sim \frac{g_{s}^{2}\left|W_{0}\right|}{\mathscr{V} \sqrt{4 \pi}} M_{p} .
$$

It is remarkable that depending on the large volume $\mathscr{V}$, the gravitino mass could be $\mathrm{TeV}$ or much larger. Considering the Kähler metric of the observable sector to have the form $\widetilde{K}_{\alpha \bar{\beta}}=$ $\widetilde{K}_{\alpha} \delta_{\alpha \bar{\beta}}$, with $\widetilde{K}_{\alpha}$ being constant, we have scalar soft masses of the following form:

$$
m_{\alpha}^{2}=m_{3 / 2}^{2},
$$

where we have neglected the very tiny cosmological constant value $V_{0}$. In the large volume limit, $\mathscr{V} \sim \tau_{5} \equiv \tau_{b} \gg \tau_{4} \equiv \tau_{s} \gg$ 1 , the Kähler metric and its inverse are given by

$$
\begin{aligned}
& K_{m \bar{n}} \simeq\left[\begin{array}{cc}
\frac{1}{\mathscr{V}} & -\frac{1}{\mathscr{V}^{5 / 3}} \\
-\frac{1}{\mathscr{V}^{5 / 3}} & \frac{1}{\mathscr{V}^{4 / 3}}
\end{array}\right], \\
& K^{m \bar{n}} \simeq\left[\begin{array}{cc}
\mathscr{V} & \mathscr{V}^{2 / 3} \\
\mathscr{V}^{2 / 3} & \mathscr{V}^{4 / 3}
\end{array}\right] .
\end{aligned}
$$

The F-terms can be calculated from

$$
F^{m}=e^{K / 2} K^{m \bar{n}} D_{\bar{n}} W .
$$

Hence, the approximate dependence of the F-terms on the volume is given by

$$
\begin{aligned}
& F^{4} \sim \frac{1}{\mathscr{V}}, \\
& F^{5} \sim \frac{1}{\mathscr{V}^{1 / 3}} .
\end{aligned}
$$

Consider a linear dependence of the gauge kinetic function on the Kähler moduli [62, 63]; therefore, the gaugino masses are given by

$$
m_{1 / 2} \sim \frac{g_{s}^{2} W_{0} M_{p}}{\sqrt{4 \pi}}\left(\frac{1}{\mathscr{V}}+\frac{1}{\mathscr{V}^{5 / 3}}\right) .
$$

Also, the universal A-term is given by

$$
A_{0} \simeq F^{m} K_{m} \sim \frac{-g_{s}^{2} W_{0} M_{p}}{\sqrt{4 \pi} \mathscr{V}} .
$$

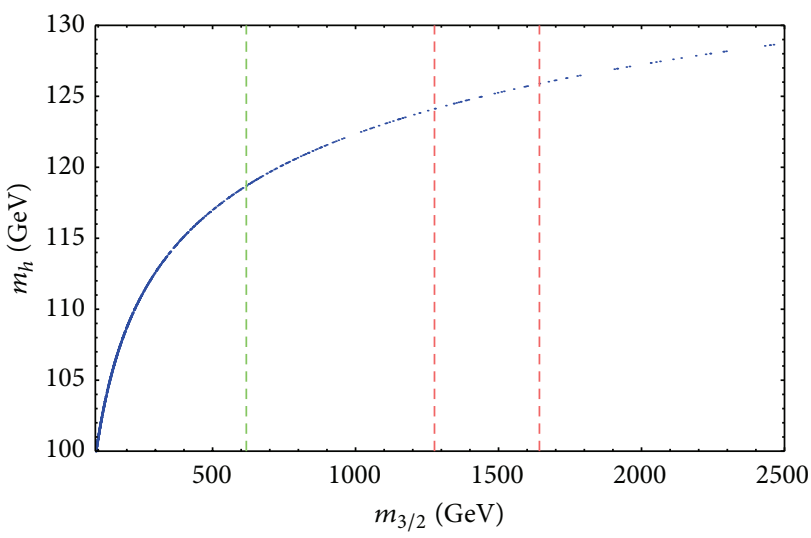

FIGURE 5: Higgs mass $m_{h}$ as a function of the gravitino mass $m_{3 / 2}$. The region in left to the green line is disallowed by the gluino mass constraint, while the area between the red lines shows the region for which the Higgs mass lies in the range of $124-126 \mathrm{GeV}$.

Accordingly, at large volumes $\mathscr{V} \sim 10^{13}-10^{15}$, the soft masses are related to the gravitino mass as follows:

$$
\begin{aligned}
& m_{0} \simeq m_{1 / 2}=m_{3 / 2}, \\
& A_{0} \simeq-m_{3 / 2} .
\end{aligned}
$$

These soft terms are generated at string scale; therefore, we have to run them to the electroweak (EW) scale and impose the EW symmetry breaking conditions to analyze the corresponding spectrum. In this case, condition (77) reads

$$
\mu^{2}+\frac{M_{Z}^{2}}{2} \simeq-0.1 m_{3 / 2}^{2}+2 m_{3 / 2}^{2},
$$

which can be satisfied easily at the $\mathrm{TeV}$ scale. The above set of soft terms is special case of CMSSM. Therefore, the Higgs mass limit requires $m_{1 / 2}$ to be of order $1.5 \mathrm{TeV}$, as shown in Figure 5, where the Higgs mass $m_{h}$ is plotted versus the gravitino mass $m_{3 / 2}$. With such heavy values of $m_{0}$ and $m_{1 / 2}$, the SUSY spectrum will be quite heavy and the relic abundance of the lightest neutralino is not consistent with Planck's results [67].

\section{Cosmological Consequences}

One of the important consequences of the moduli stabilization is its effect on inflation. It turned out that the scalar potential of the modulus field in KKLT is not suitable to account for single-field inflation since the modulus potential is not flat enough to allow for slow roll $[68,69]$. Namely, the real part is not protected by a shift symmetry which will result in an $\eta$-problem. Although the axionic part is subjected to shift symmetry, the field dependent mass matrix is not diagonal with a significant mixing. Therefore, the shift symmetry is violated and the $\eta$-problem persists (for a review for string inflation in the light of recent observations, see, e.g., [70]).

In this section, we discuss how this problem can be evaded in what is called the racetrack superpotential. Also, 


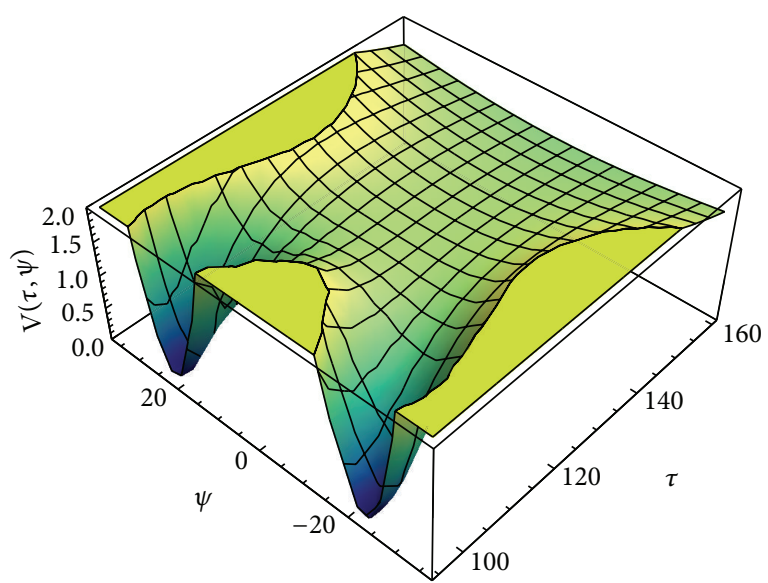

(a)

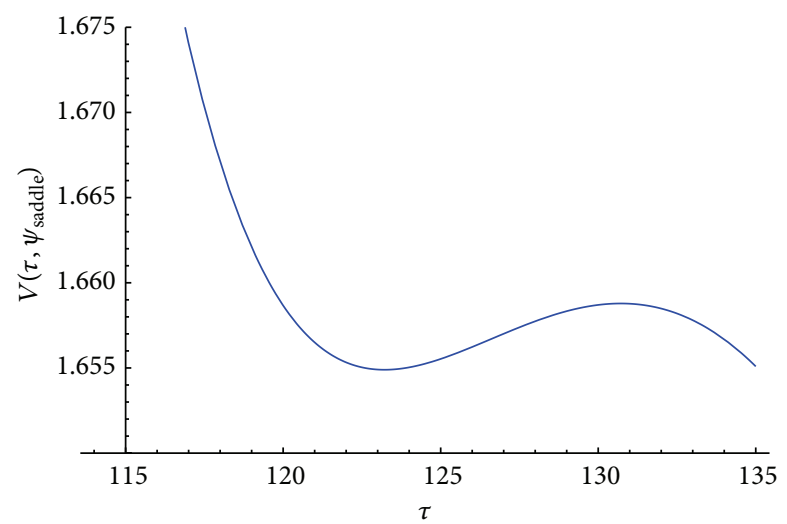

(b)

FIGURE 6: (a) corresponds to the racetrack scalar potential and (b) corresponds to the variation of the potential in the neighborhood of the saddle point (all are multiplied by $10^{16}$ ). The parameter values are $A=0.02, B=0.035, a=2 \pi / 100, b=2 \pi / 90, E_{2}=4.14668 \times 10^{-12}$, and $W_{0}=-4 \times 10^{-5}$.

inflation via Kähler moduli is discussed. In addition, we explain the destabilization problem that arises in KKLT and LVS. Finally, we study the effect of moduli backreaction on inflationary scenarios.

6.1. Racetrack Inflationary Model. In the racetrack model, the superpotential is given by $[68,69]$

$$
W=W_{0}+A e^{-a T}+B e^{-b T},
$$

where the additional nonperturbative term may be obtained from gaugino condensation in a theory with a product of gauge groups such as $S U(N) \times S U(M)$ gauge groups; hence, $a=2 \pi / M$ and $b=2 \pi / N$. This is known as the racetrack model. In this case, the global SUSY minimum is located at

$$
T_{0}=\frac{M N}{M-N} \log \left(-\frac{M B}{N A}\right) .
$$

The inflationary potential is given by the F-term scalar potential added to the uplifting term as follows:

$$
\begin{aligned}
V_{\text {inf }} & =\frac{E_{2}}{\tau^{2}}+\frac{e^{-4 \tau(a+b)}}{6 \tau^{2}}\left\{a A^{2}(a \tau+3) e^{2 \tau(a+2 b)}\right. \\
& +e^{3 \tau(a+b)}[A B(2 a b \tau+3(a+b))(\cos (\tau(a-b))) \\
& \left.+3 a A W_{0} e^{b \tau} \cos (a \psi)+3 b B W_{0} e^{a \tau} \cos (b \psi)\right] \\
& \left.+b B^{2}(b \tau+3) e^{2 \tau(2 a+b)}\right\} .
\end{aligned}
$$

Figure 6 shows the scalar potential with two degenerate minima and one saddle point located at $\psi_{\text {saddle }}=0$. At this saddle point, the potential has a minimum in the $\tau$ direction and a maximum in the $\psi$ direction. The inflaton is considered to be rolling slowly from initial conditions near the saddle point on the inflationary trajectory. Namely, the initial motion is in $\psi$ direction and the inflationary path is determined numerically $[68,69]$.
The racetrack inflation model predicts spectral index $n_{s}=$ 0.96 , inflation scale $M_{\text {inf }} \sim 10^{14} \mathrm{GeV}$, and tiny tensor to scalar ratio $r \simeq\left(M_{\mathrm{inf}} / M_{\mathrm{GUT}}\right)^{4} \sim 10^{-8}$.

6.2. Kähler Moduli Inflation in the LVS. The idea of Kähler moduli inflation [71] is to produce an inflationary potential similar to the form

$$
V_{\text {inf }}(\varphi)=V_{0}\left(1-k \beta e^{-k \varphi}\right),
$$

where $\beta$ and $k$ are positive constants.

In the case of multimoduli Calabi-Yau geometries, the Calabi-Yau volume can take the form

$$
\mathscr{V}=\frac{\alpha}{2 \sqrt{2}}\left[(T+\bar{T})^{3 / 2}-\sum_{j=1}^{n} \lambda_{j}\left(T_{j}+\bar{T}_{j}\right)^{3 / 2}\right],
$$

where $T=\tau+i \psi$ is responsible for the large volume and $T_{j}=\tau_{j}+i \psi_{j}$, where $\tau_{j}$ are the blow-ups. Here, $\alpha$ and $\lambda_{j}$ are model dependent positive constants. In this case, the Kähler potential is given by

$$
K=K_{\mathrm{cs}}-2 \ln \left[\mathscr{V}+\frac{\xi}{2}\right]
$$

while the superpotential is given by

$$
W=W_{0}+\sum_{i=2}^{n} A_{i} e^{-a_{i} T_{i}}
$$

where $a_{i}=2 \pi / g_{s} N$. The minimum of the scalar potential exists at the large volume limit $\mathscr{V} \sim \tau \gg \tau_{j}$ and the scalar potential has the form [71]

$$
\begin{aligned}
V= & \sum_{i} \frac{8\left(a_{i} A_{i}\right)^{2} \sqrt{\tau_{i}}}{3 \mathscr{V} \lambda_{i} \alpha} e^{-2 a_{i} \tau_{i}}-\sum_{i} 4 \frac{a_{i} A_{i}}{\mathscr{V}^{2}} W_{0} \tau_{i} e^{-a_{i} \tau_{i}} \\
& +\frac{3 \xi W_{0}^{2}}{4 \mathscr{V}^{3}} .
\end{aligned}
$$


The inflation can occur away from the minimum and the inflaton is considered to be one of the small moduli $\tau_{n}$. The volume $\mathscr{V}$ and the small moduli, other than the inflaton, are guaranteed to be stabilized to their minima during the inflation [71]. Therefore, the inflationary potential is given by [71]

$$
V_{\mathrm{inf}}=V_{0}-\frac{4 a_{n} A_{n} W_{0} \tau_{n} e^{-k \varphi}}{\mathscr{V}^{2}}
$$

with $V_{0}=\beta W_{0} / \mathscr{V}^{3}$ which is constant during the inflation. This model predicts $0,967>n_{s}>0.960$ and $r \sim 10^{-10}$ for the number of $e$-folding $50<N_{e}<60$ with inflationary scale $M_{\text {inf }} \sim 10^{13} \mathrm{GeV}$.

6.3. Destabilization Problem. In the KKLT scenario, the modulus mass is given by [36]

$$
m_{\tau}^{2}=\left.\frac{V^{\prime \prime}(\tau)}{2 K_{T \bar{T}}}\right|_{\tau_{0}}=\left.\frac{2}{9}\left(D_{T} W\right)_{\tau}\left(\tau W_{T T}-2 W_{T}\right)\right|_{\tau_{0}} .
$$

Therefore, it is linked to $m_{3 / 2}$ by the relation

$$
m_{\tau}=\left.\frac{\sqrt{2 \tau}}{9} W_{T T}\right|_{\tau_{0}}=2 a \tau_{0} m_{3 / 2}
$$

which is approximately two orders of magnitude greater than the $\mathrm{TeV}$ scale gravitino mass. In case there is an inflaton field different from modulus field with mass $\sim 10^{13} \mathrm{GeV}$, the modulus may perturb GUT scale inflation. It turns out that a problem arises due to the conflict between the requirement of a high energy scale (GUT scale) inflationary phenomenon and low energy ( $\mathrm{TeV}$ scale) SUSY phenomenology in the context of KKLT. The conflict is stemming from the constraint $[11,12]$

$$
m_{3 / 2} \gtrsim H,
$$

where $H$ is the Hubble parameter. This constraint originates from the fact that the barrier $V_{B}$ from the runaway direction, as exhibited in Figure 7, is a bit less than the magnitude of the AdS minimum $\left|V_{0}^{\text {AdS }}\right|$ since the uplift effect is negligible for large $\tau$. Hence, we have

$$
V_{B} \lesssim V_{0}^{\mathrm{AdS}} \simeq 3 m_{3 / 2}^{2} .
$$

Therefore, recovering the Planck mass, we have

$$
V_{B} \sim m_{3 / 2}^{2} M_{p}^{2} .
$$

If we included an inflaton, there will be a contribution to the overall inflationary potential by a term of the form $V(\varphi) / \tau^{n}$ due to the fact that the modulus couples to all sources of energy. Accordingly, the total inflationary potential at the KKLT dS minimum is given by

$$
V_{\mathrm{inf}}=V_{\mathrm{dS}}(\tau)+\frac{V(\varphi)}{\tau^{n}} \simeq \frac{V(\varphi)}{\tau^{n}} .
$$

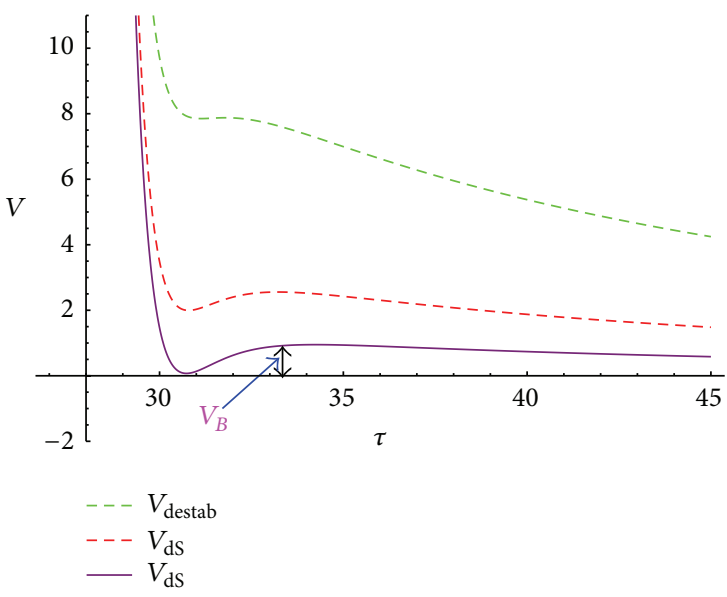

FIGURE 7: The purple curve shows the dS vacuum, while the green one displays the destabilization of the modulus due to large contribution of vacuum energy density.

In this case, there will be a competition between the runaway dependence $\tau^{-n}$ and the barrier $V_{B}$. Therefore, protecting the volume modulus from destabilization will impose the condition

$$
\begin{aligned}
V_{\text {inf }} & \lesssim V_{B} \sim 3 m_{3 / 2}^{2} \Longrightarrow \\
m_{3 / 2} & \gtrsim H .
\end{aligned}
$$

On the other hand, in the LVS case, there is an extra suppression by large volume $\mathscr{V}$ [72]

$$
V_{B} \sim \frac{m_{3 / 2}^{2} M_{p}^{2}}{\mathscr{V}} \sim m_{3 / 2}^{3} M_{p}
$$

and correspondingly we have the constraint

$$
H \lesssim m_{3 / 2}^{3 / 2},
$$

which will not improve the situation much. In this regard, $\mathrm{TeV}$ scale gravitino mass which is favored by low energy phenomenology implies disfavored nontraditional low scale inflation. The property of $H \leqslant m_{3 / 2}$ seems to be a common property between many inflationary models in stabilization scenarios in string theories [72-74]. This problem is sometimes called the Kallosh-Linde problem. The latter originates from the fact that compatible large scale models of inflation require a very large gravitino mass, where

$$
m_{\varphi} \ll H \leqslant m_{3 / 2} .
$$

The KL Model and Strong Moduli Stabilization. The problem of destabilization can be evaded in models of strong moduli stabilization like the KL model $[11,12]$. In this scenario, the volume is still determined by one modulus $T$, but there is an additional nonperturbative term contributing to the racetrack superpotential

$$
W=W_{0}+A e^{-a T}-B e^{-b T} .
$$


In this respect, the imaginary part stabilizes at the origin again, whereas the potential of the real part $\tau$ is given by

$$
V(\tau)=\frac{e^{-2 \tau(a+b)}\left(a A e^{b \tau}-b B e^{a \tau}\right)\left(e^{b \tau}\left(A(a \tau+3)+3 w 0 e^{a \tau}\right)-B e^{a \tau}(b \tau+3)\right)}{6 \tau^{2}}
$$

This potential possesses two minima; one is a metastable supersymmetric Minkowski vacuum at

$$
\tau_{0}=\frac{1}{a-b} \ln \left(\frac{a A}{b B}\right),
$$

and the other is a deeper AdS one as shown in Figure 8. The $\mathrm{KL}$ modulus squared mass is given by [36]

$$
m_{\tau}^{2}=\frac{2 a A b B(a-b)}{9}\left(\frac{a A}{b B}\right)^{-(a+b) /(a-b)} \ln \left(\frac{a A}{b B}\right) .
$$

Therefore, for $A=B=1, a=0.1$, and $b=0.05$, we find $m_{\tau} \sim 4 \times 10^{15} \mathrm{GeV}$ which means that the volume modulus mass is much larger than the inflaton mass $\left(m_{\varphi} \sim\right.$ $10^{13} \mathrm{GeV}$ ) and accordingly will be frozen quickly during the inflation without perturbing the inflaton dynamics. Clearly, this is much featured than the KKLT case since the gravitino mass vanishes at the SUSY minimum. Therefore, the hight of the barrier from the runaway direction is independent of the gravitino mass; hence, the Hubble parameter is also independent of the gravitino mass. In this case, KL model can account for high scale inflation.

In order to obtain interesting phenomenology at the $\mathrm{TeV}$ scale for SUSY breaking without conflict with the high scale inflation requirements, a constant shift $\Delta W$ is added to the superpotential [36] and it is supposed to be of the order of the weak scale. In this case, the value of $\tau$ minimizing the potential will be shifted to $\tau_{0}+\delta \tau$. The SUSY vacuum is obtained by solving the equation of motion

$$
D_{T} W\left(\tau_{0}+\delta \tau\right)=0,
$$

which implies

$$
\delta \tau=\frac{3 \Delta W}{2 \tau_{0} W_{T, T}\left(\tau_{0}\right)} .
$$

Correspondingly, the AdS minimum is independent of the sign of $\Delta W$ and is approximated to

$$
V_{0}^{\mathrm{AdS}} \simeq-\frac{3(\Delta W)^{2}}{8 \tau_{0}^{3}}
$$

where the above expressions are obtained using $W_{T}\left(\tau_{0}\right)=0$, $W\left(\tau_{0}\right)=\Delta W$, and $W_{T}\left(\tau_{0}+\delta \tau\right)=\delta \tau W_{T, T}\left(\tau_{0}\right)$. The AdS vacuum can be uplifted to a dS one by mechanisms similar to those in the KKLT model and hence SUSY will be broken with the gravitino becoming massive. In this respect, the gravitino mass is given in terms of $\Delta W$ as follows:

$$
m_{3 / 2} \simeq \sqrt{\frac{\left|V_{0}^{\text {AdS }}\right|}{3}}=\frac{1}{2 \sqrt{2}}\left(\frac{a-b}{\ln (a A / b B)}\right)^{3 / 2}|\Delta W| .
$$

It is worth mentioning that the uplifting effect here is so small and cannot exceed the barrier between the dS and the AdS vacua which is one of the strengths of this model. On the other hand, a weakness of the approach is the lack of interpretation of the origin of the scale of the shift $\Delta W$ which should be around $10^{-13}$, if we are seeking TeV scale gravitino mass.

6.4. Impact of String Moduli Backreaction. Here, we give a brief overview of the effect of string moduli backreaction on the inflationary models and its link to SUSY breaking. To study such effects on the inflation, models of strong moduli stabilization are favored such as the KL model. In the latter model, the moduli are heavy; in particular, the modulus mass is larger than the Hubble parameter. The moduli backreaction effect on the inflation and its link to SUSY breaking were studied in many research papers [7579]. For a single modulus field case, the impact of the stabilized volume modulus field on large and small field inflation was studied in [75]. In this respect, the total Kähler potential and superpotential are given by

$$
\begin{aligned}
K & =K_{\bmod }(T+\bar{T})+K_{\mathrm{inf}}\left(\phi_{\alpha}, \bar{\phi}_{\bar{\alpha}}\right) \\
& =-\kappa \log (T+\bar{T})+K_{\mathrm{inf}}, \\
W & =W_{\mathrm{mod}}(T)+W_{\mathrm{inf}}\left(\phi_{\alpha}\right),
\end{aligned}
$$

where $\phi_{\alpha}$ are chiral superfields related to the inflationary scenario and the constant $\kappa=1$ for heterotic dilaton and $\kappa=3$ for type IIB Kähler modulus. Accordingly, the F-term scalar potential can be written as

$$
V=e^{K_{\text {inf }}} V_{\text {mod }}(T)+(2 \tau)^{-\kappa} V_{\text {inf }}\left(\phi_{\alpha}\right)+e^{K} \widetilde{V}\left(T, \phi_{\alpha}\right),
$$

where

$$
\begin{aligned}
V_{\text {mod }} & =e^{K_{\text {mod }}}\left[K^{T \bar{T}}\left|D_{T} W_{\text {mod }}\right|^{2}-3\left|W_{\text {mod }}\right|^{2}\right], \\
V_{\text {inf }} & =e^{K_{\text {inf }}}\left[K^{\alpha \bar{\alpha}}\left|D_{\alpha} W_{\text {inf }}\right|^{2}-3\left|W_{\text {inf }}\right|^{2}\right] .
\end{aligned}
$$

In this case, the potential is minimized at $\tau_{0}+\delta T$, due to the effect of the inflationary large positive energy density, where $\tau_{0}$ corresponds to the SUSY Minkowski minimum, if the scenario contains moduli only. Since the modulus is very heavy, it stabilizes quickly to its minimum and 


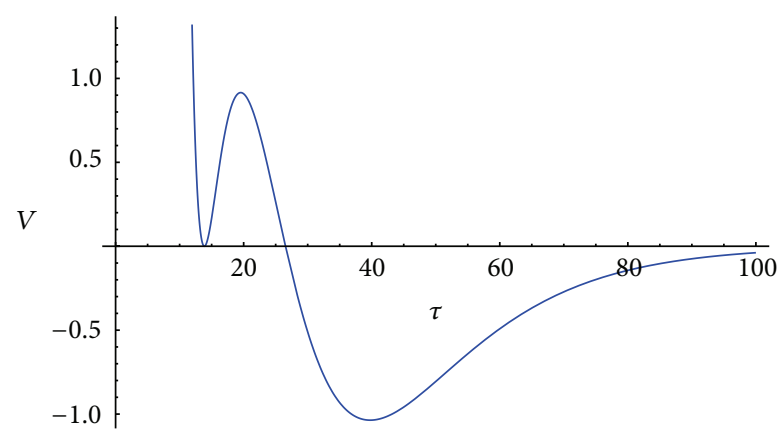

FIgURE 8: The F-term scalar potential (multiplied by $10^{7}$ ) for KL model possesses both metastable Minkowski vacuum which is supersymmetric and another AdS minimum, for $A=B=1, a=0.1$, and $b=0.05$.

the inflationary potential gets corrections after setting $T$ to its minimum as follows [75]:

$$
\begin{aligned}
V & =\left(2 \tau_{0}\right)^{-3} V_{\mathrm{inf}}-\frac{\kappa}{2\left(2 \tau_{0}\right)^{3 \kappa / 3} m_{T}}\left\{W _ { \mathrm { inf } } \left[V_{\mathrm{inf}}\right.\right. \\
& \left.\left.+e^{K_{\mathrm{inf}}} K^{\alpha \bar{\alpha}} \partial_{\alpha} W_{\mathrm{inf}} D_{\bar{\alpha}} \bar{W}_{\mathrm{inf}}\right]+ \text { h.c. }\right\} \\
& -\frac{\kappa e^{K_{\mathrm{inf}}}}{\left(2 \tau_{0}\right)^{2 \kappa} m_{T}^{2}}\left|K^{\alpha \bar{\alpha}} D_{\alpha} W_{\mathrm{inf}} \partial_{\bar{\alpha}} \bar{W}_{\mathrm{inf}}\right| .
\end{aligned}
$$

Now, consider a case of small field inflation such as the F-term Hybrid inflation [80, 81], where the Kähler potential and superpotential are given by

$$
\begin{gathered}
K_{\mathrm{inf}}=|\varphi|^{2}+\left|\phi_{1}\right|^{2}+\left|\phi_{2}\right|^{2}, \\
W=\lambda \varphi\left(M^{2}-\phi_{1} \phi_{2}\right),
\end{gathered}
$$

where $\varphi$ is the inflaton and $\phi_{1}, \phi_{2}$ correspond to the waterfall fields. The hybrid inflation scenario supposes that, for values of $\varphi>\varphi_{c}=M$, the potential is minimized at $\phi_{1}=\phi_{2}=0$ and, therefore, the inflationary superpotential is effectively given by

$$
W_{\text {inf }}=\lambda M^{2} \varphi
$$

Accordingly, the corrected inflationary potential due to the moduli backreaction will contain a linear term in the inflaton [75]

$$
V_{\text {inf }}(\varphi)=V_{0}\left[1-\frac{2 \kappa \sqrt{V_{0}}}{m_{T}} \operatorname{Re}(\varphi)\right]+\Delta V_{1}+V_{\text {sug }},
$$

where $\Delta V_{1}$ is the one-loop correction to the inflation potential and $V_{\text {sug }}$ represents the contribution due to supergravity, which has small effect and can be neglected. Here, $V_{0}$ is the rescaled vacuum energy during inflation given by $V_{0}=\tilde{\lambda}^{2} M^{4}$, with $\tilde{\lambda}^{2}=\lambda^{2} /\left(2 \tau_{0}\right)^{\kappa}$. In this case, the value of the spectral index can be improved; $n_{s} \simeq 0.96$, compared to its value in the pure hybrid scenarios; $n_{s}=0.98$. So, we do not need to go to nonminimal Kähler scenario [80, 81].
Another example is the large field inflation which was successfully embedded in supergravity. In this case, a shift symmetry is used to avoid the $\eta$-problem as well as considering stabilizer field $S$ [82-87]. In this regard, consider a form of the Kähler potential and superpotential given by

$$
\begin{aligned}
& K_{\text {inf }}=-\frac{1}{2}|\varphi-\bar{\varphi}|^{2}+|S|^{2}-\zeta|S|^{4}, \\
& W_{\text {inf }}=S f(\varphi),
\end{aligned}
$$

where the quartic term in the Kähler potential causes stabilizer $S$ to get mass much larger than the Hubble parameter and so stabilizes quickly to the origin. The Kähler potential is independent of the real part $\operatorname{Re}(\varphi)=\varphi_{r}$, due to the shift symmetry and, hence, it will correspond to the inflaton. The $S$ field and the imaginary part of $\varphi$ will be stabilized at zero and hence the inflationary potential (which is given in pure inflation scenario case by $\left.V\left(\varphi_{r}\right)=\left|f\left(\varphi_{r}\right)\right|^{2}\right)$ get corrections as follows [75]:

$$
V\left(\varphi_{r}\right)=\widetilde{V}\left[1-\kappa \frac{\widetilde{V}}{m_{T}^{2}}-\kappa^{2} \frac{\widetilde{V}^{2}}{m_{T}^{2} m_{S}^{2}}\right],
$$

where $\widetilde{V}\left(\varphi_{r}\right)=\left|f\left(\varphi_{r}\right)\right|^{2} /\left(2 \tau_{0}\right)^{\kappa}$. For $f(\varphi)=m \varphi$, we get the case of chaotic inflation. It is worth noting that the leading correction term here is proportional to $1 / \mathrm{m}_{T}^{2}$, whereas in the case of hybrid inflation it is proportional to $1 / m_{T}$.

\section{Conclusions}

In this minireview, we have analyzed the problem of moduli stabilization in type IIB string theory with positive vacuum energy. We focused on KKLT and Large Volume Scenarios, where geometrical fluxes and nonperturbative superpotentials are required to stabilize complex structure moduli, dilation, and Kähler moduli. We also discussed some possible mechanisms for uplifting the AdS minimum and making it a metastable de Sitter ground state. We have derived the soft SUSY breaking terms in these models. We showed that in KKLT these terms are not consistent with electroweak breaking conditions and hence they are not phenomenologically viable, while in LVS we found that the scalar masses and gaugino masses and trilinear terms are universal and are given in terms of gravitino mass $m_{3 / 2}$. We emphasized that a very heavy spectrum with $m_{3 / 2} \sim$ $1.5 \mathrm{TeV}$ is required to account for the lightest Higgs mass limit. However, in this case, the relic abundance of the lightest neutralino is not consistent with the measured limits. We also studied inflation scenarios associated with the moduli stabilization. We considered two examples of racetrack and Kähler inflation. Finally, we commented on the problem of moduli destabilization and moduli backreaction effects on inflation.

The recent developments in the KKLT and the LVS are worth mentioning. One of the updates is the anti-D3-brane uplift via a nilpotent superfield [88]. In the case of the LVS, the universal gaugino masses are of the same order as the scalar masses and have lower limits $\sim 10^{3} \mathrm{TeV}$ implied from the 
cosmological moduli problem. This will imply heavy SUSY spectrum which is far from the current LHC detectability. A similar situation for the KKLT model holds. Another modification is the sequestered scenario analyzed in [65]. In this scenario, the TeV scale MSSM and split SUSY model are obtained. For the MSSM scenario, $M_{1 / 2} \sim m_{0} \ll m_{3 / 2}$. For split SUSY scenario, $M_{1 / 2} \ll m_{0} \ll m_{3 / 2}$.

\section{Conflict of Interests}

The authors declare that there is no conflict of interests regarding the publication of this paper.

\section{Acknowledgments}

This work was partially supported by the STDF Project 13858 and the ICTP Grant AC-80. Ahmad Moursy would like to thank W. Abdallah for the useful discussions.

\section{References}

[1] A. D. Dolgov, "Long-range forces in the universe," Physics Report, vol. 320, no. 1-6, pp. 1-15, 1999.

[2] P. Candelas, G. T. Horowitz, A. Strominger, and E. Witten, "Vacuum configurations for superstrings," Nuclear Physics B, vol. 258, no. 1, pp. 46-74, 1985.

[3] F. Denef, "Les Houches lectures on constructing stringvacua," http://arxiv.org/abs/0803.1194.

[4] A. Strominger and E. Witten, "New manifolds for superstring compactification," Communications in Mathematical Physics, vol. 101, no. 3, pp. 341-361, 1985.

[5] A. Strominger, "Superstrings with torsion," Nuclear Physics. B, vol. 274, no. 2, pp. 253-284, 1986.

[6] E. Witten, "New issues in manifolds of SU(3) holonomy," Nuclear Physics B, vol. 268, no. 1, pp. 79-112, 1986.

[7] S. Kachru, R. Kallosh, A. Linde, and S. P. Trivedi, "de Sitter vacua in string theory," Physical Review D, vol. 68, no. 4, Article ID 046005, 10 pages, 2003.

[8] V. Balasubramanian, P. Berglund, J. P. Conlon, and F. Quevedo, "Systematics of moduli stabilisation in Calabi-Yau flux compactifications," Journal of High Energy Physics, vol. 2005, no. 3, article 007, 22 pages, 2005.

[9] V. Balasubramanian, P. Berglund, J. P. Conlon, and F. Quevedo, "Systematics of moduli stabilisation in Calabi-Yau flux compactifications," Journal of High Energy Physics, vol. 2005, no. 3, article 007, 2005.

[10] J. P. Conlon, F. Quevedo, and K. Suruliz, "Large-volume flux compactifications: moduli spectrum and D3/D7 soft supersymmetry breaking," Journal of High Energy Physics, vol. 2005, no. 8, article 007, 2005.

[11] R. Kallosh and A. D. Linde, "Landscape, the scale of SUSY breaking, and inflation," Journal of High Energy Physics, vol. 2004, no. 12, article 004, 2004.

[12] R. Kallosh and A. D. Linde, "Testing string theory with CMB," Journal of Cosmology and Astroparticle Physics, vol. 2007, no. 4, article 017, 2007.

[13] O. Lebedev, H. P. Nilles, and M. Ratz, "de Sitter vacua from matter superpotentials," Physics Letters B, vol. 636, no. 2, pp. 126-131, 2006.
[14] N. Seiberg, "Naturalness versus supersymmetric nonrenormalization theorems," Physics Letters. B, vol. 318, no. 3, pp. 469-475, 1993.

[15] M. Dine and N. Seiberg, "Nonrenormalization theorems in superstring theory," Physical Review Letters, vol. 57, no. 21, pp. 2625-2628, 1986.

[16] M. Dine, R. Rohm, N. Seiberg, and E. Witten, "Gluino condensation in superstring models," Physics Letters B, vol. 156, no. 1-2, pp. 55-60, 1985.

[17] L. J. Dixon and J. A. Harvey, "String theories in ten dimensions without spacetime supersymmetry," Nuclear Physics B, vol. 274, no. 1, pp. 93-105, 1986.

[18] L. Alvarez-Gaume, P. H. Ginsparg, G. W. Moore, and C. Vafa, "An $\mathrm{O}(16) \times \mathrm{O}(16)$ heterotic string," Physics Letters B, vol. 171, no. 2-3, pp. 155-162, 1986.

[19] V. P. Nair, A. Shapere, A. Strominger, and F. Wilczek, "Compactification of the twisted heterotic string," Nuclear Physics B, vol. 287, no. 3, pp. 402-418, 1987.

[20] J. Scherk and J. H. Schwarz, "How to get masses from extra dimensions," Nuclear Physics B, vol. 153, no. 1-2, pp. 61-88, 1979.

[21] J. Polchinski, String Theory, Cambridge University Press, Cambridge, UK, 1998.

[22] S. B. Giddings, S. Kachru, and J. Polchinski, "Hierarchies from fluxes in string compactifications," Physical Review D, vol. 66, no. 10, Article ID 106006, 16 pages, 2002.

[23] J. M. Maldacena and C. Nuñez, "Supergravity description of field theories on curved manifolds and a no go theorem," International Journal of Modern Physics A, vol. 16, no. 5, pp. 822$855,2001$.

[24] B. de Wit, D. J. Smit, and N. D. Hari Dass, "Residual supersymmetry of compactified D $=10$ supergravity," Nuclear Physics B, vol. 283, pp. 165-191, 1987.

[25] M. R. Douglas and S. Kachru, "Flux compactification," Reviews of Modern Physics, vol. 79, no. 2, pp. 733-796, 2007.

[26] M. Graña, "Flux compactifications in string theory: a comprehensive review," Physics Reports, vol. 423, no. 3, pp. 91-158, 2006.

[27] F. Denef, M. R. Douglas, and S. Kachru, "Physics of string flux compactifications," Annual Review of Nuclear and Particle Science, vol. 57, pp. 119-144, 2007.

[28] R. Blumenhagen, B. Kors, D. Lust, and S. Stieberger, "Fourdimensional string compactifications with D-branes, orientifolds and fluxes," Physics Reports, vol. 445, no. 1-6, pp. 1-193, 2007.

[29] J. Wess and J. Bagger, Supersymmetry and Supergravity, Princeton Series in Physics, Princeton University Press, Princeton, NJ, USA, 2nd edition, 1992.

[30] D. Bailin and A. Love, Supersymmetric Gauge Field Theory and String Theory, Graduate Student Series in Physics, IOP, Bristol, UK, 1994.

[31] L. E. Ibanez and A. M. Uranga, String Theory and Particle Physics: An Introduction to String Phenomenology, Cambridge University Press, Cambridge, UK, 2012.

[32] S. Gukov, C. Vafa, and E. Witten, "CFT's from Calabi-Yau fourfolds," Nuclear Physics B, vol. 584, no. 1-2, pp. 69-108, 2000, Erratum in: Nuclear Physics B, vol. 608, no. 1-2, pp. 477-478, 2001.

[33] E. Cremmer, S. Ferrara, C. Kounnas, and D. V. Nanopoulos, "Naturally vanishing cosmological constant in $N=1$ supergravity," Physics Letters B, vol. 133, no. 1-2, pp. 61-66, 1983. 
[34] J. R. Ellis, A. B. Lahanas, D. V. Nanopoulos, and K. Tamvakis, "No-scale supersymmetric standard model," Physics Letters B, vol. 134, no. 6, pp. 429-435, 1984.

[35] R. Barbieri, E. Cremmer, and S. Ferrara, "Flat and positive potentials in $N=1$ supergravity," Physics Letters B, vol. 163, no. 1-4, pp. 143-147, 1985.

[36] A. Linde, Y. Mambrini, and K. A. Olive, "Supersymmetry breaking due to moduli stabilization in string theory," Physical Review D, vol. 85, no. 6, Article ID 066005, 2012.

[37] C. P. Burgess, R. Kallosh, and F. Quevedo, "De Sitter string vacua from supersymmetric D-terms," Journal of High Energy Physics, vol. 2013, no. 10, article 056, 14 pages, 2003.

[38] G. Villadoro and F. Zwirner, "de Sitter vacua via consistent $D$ terms," Physical Review Letters, vol. 95, no. 23, Article ID 231602, 2005.

[39] A. Achúcarro, B. de Carlos, J. A. Casas, and L. Doplicher, "de Sitter vacua from uplifting D-terms in effective supergravities from realistic strings," Journal of High Energy Physics, vol. 2006, no. 6, article 014, 2006.

[40] K. Choi and K.-S. Jeong, "Supersymmetry breaking and moduli stabilization with anomalous U(1) gauge symmetry," Journal of High Energy Physics, vol. 2006, no. 8, article 007, 2006.

[41] E. Dudas and Y. Mambrini, "Moduli stabilization with positive vacuum energy," Journal of High Energy Physics, vol. 2006, no. 10, article 044, 2006.

[42] M. Haack, D. Krefl, D. Lüst, A. Van Proeyen, and M. Zagermann, "Gaugino condensates and D-terms from D7-branes," Journal of High Energy Physics, vol. 2007, no. 1, article 078, 2007.

[43] C. P. Burgess, J. M. Cline, K. Dasgupta, and H. Firouzjahi, "Uplifting and inflation with D3 branes," Journal of High Energy Physics, vol. 2007, no. 3, article 027, 2007.

[44] R. Kallosh and A. D. Linde, "O'KKLT," Journal of High Energy Physics, vol. 2007, no. 2, article 002, 13 pages, 2007.

[45] A. Westphal, "De Sitter string vacua from Kähler uplifting," Journal of High Energy Physics, vol. 2007, no. 3, article 102, 2007.

[46] M. Rummel and A. Westphal, "A sufficient condition for de Sitter vacua in type IIB string theory," Journal of High Energy Physics, vol. 2012, no. 1, article 020, 44 pages, 2012.

[47] J. Louis, M. Rummel, R. Valandro, and A. Westphal, "Building an explicit de Sitter," Journal of High Energy Physics, vol. 2012, no. 10, article 163, 2012.

[48] A. Awad, N. Chamoun, and S. Khalil, "On flux compactification and moduli stabilization," Physics Letters B, vol. 635, no. 2-3, pp. 136-140, 2006.

[49] L. E. Ibáñez, C. Muñoz, and S. Rigolin, "Aspects of type I string phenomenology," Nuclear Physics B, vol. 553, no. 1-2, pp. 43-80, 1999.

[50] V. Balasubramanian and P. Berglund, "Stringy corrections to Kahler potentials, SUSY breaking, and the cosmological constant problem," Journal of High Energy Physics, vol. 2004, no. 11, article 085, 2004.

[51] K. Becker, M. Becker, M. Haack, and J. Louis, "Supersymmetry breaking and alpha-prime corrections to flux induced potentials," Journal of High Energy Physics, vol. 2002, no. 6, article 060, 2002.

[52] T. W. Grimm and J. Louis, "The effective action of N = 1 CalabiYau orientifolds," Nuclear Physics B, vol. 699, no. 1-2, pp. 387426, 2004.

[53] A. Brignole, L. E. Ibáñez, and C. Muñoz, "Soft supersymmetry breaking terms from supergravity and superstring models," in
Perspectives on Supersymmetry II, vol. 21 of Advanced Series on Directions in High Energy Physics, pp. 244-268, World Scientific, 2010.

[54] D. G. Cerdeño and C. Muñoz, "An introduction to supergravity," in Proceedings of the Corfu Summer Institute on Elementary Particle Physics (PoS CORFU '98), p. 11, Kerkyra, Greece, September 1998.

[55] K. Choi, A. Falkowski, H. P. Nilles, and M. Olechowski, "Soft supersymmetry breaking in KKLT flux compactification," Nuclear Physics B, vol. 718, no. 1-2, pp. 113-133, 2005.

[56] K. Choi, K.-S. Jeong, and K.-I. Okumura, "Phenomenology of mixed modulus-anomaly mediation in fluxed string compactifications and brane models," Journal of High Energy Physics, vol. 2005, no. 9, article 039, 2005.

[57] M. Endo, M. Yamaguchi, and K. Yoshioka, "A bottom-up approach to moduli dynamics in heavy gravitino scenario: superpotential, soft terms and sparticle mass spectrum," Physical Review D, vol. 72, no. 1, Article ID 015004, 2005.

[58] A. Falkowski, O. Lebedev, and Y. Mambrini, "SUSY phenomenology of KKLT flux compactifications," Journal of High Energy Physics, vol. 2005, no. 11, article 034, 27 pages, 2005.

[59] O. Lebedev, V. Löwen, H. P. Nilles, Y. Mambrini, and M. Ratz, "Metastable vacua in flux compactifications and their phenomenology," Journal of High Energy Physics, vol. 2007, no. 2, article 063, 23 pages, 2007.

[60] B. C. Allanach, F. Quevedo, and K. Suruliz, "Low-energy supersymmetry breaking from string flux compactifications: benchmark scenarios," Journal of High Energy Physics, vol. 2006, no. 4 , article $040,2006$.

[61] J. P. Conlon and F. Quevedo, "Gaugino and scalar masses in the landscape," Journal of High Energy Physics, vol. 2006, no. 6, article 029, 17 pages, 2006.

[62] J. P. Conlon, S. S. Abdussalam, F. Quevedo, and K. Suruliz, "Soft SUSY breaking terms for chiral matter in IIB string compactifications," Journal of High Energy Physics, vol. 2007, no. 1, article 032, 2007.

[63] J. P. Conlon, D. Cremades, and F. Quevedo, "Kähler potentials of chiral matter fields for Calabi-Yau string compactifications," Journal of High Energy Physics, vol. 2007, no. 1, article 022, 2007.

[64] S. Angus and J. P. Conlon, "Soft supersymmetry breaking in anisotropic LARGE volume compactifications," Journal of High Energy Physics, vol. 2013, no. 3, article 071, 2013.

[65] L. Aparicio, M. Cicoli, S. Krippendorf, A. Maharana, F. Muia, and F. Quevedo, "Sequestered de Sitter string scenarios: softterms," Journal of High Energy Physics, vol. 2014, no. 11, article 071, 2014.

[66] M. Chakraborti, U. Chattopadhyay, S. Rao, and D. P. Roy, "Higgsino dark matter in nonuniversal gaugino mass models," Physical Review D, vol. 91, no. 3, Article ID 035022, 2015.

[67] W. Abdallah and S. Khalil, "MSSM dark matter in light of Higgs and LUX results," Advances in High Energy Physics, vol. 2016, Article ID 5687463, 10 pages, 2016.

[68] J. J. Blanco-Pillado, C. P. Burgess, J. M. Cline et al., "Racetrack inflation," Journal of High Energy Physics, vol. 2004, no. 11, article 063, 2004.

[69] J. J. Blanco-Pillado, C. P. Burgess, J. M. Cline et al., "Inflating in a better racetrack," Journal of High Energy Physics, vol. 2006, no. 9, article 002, 22 pages, 2006.

[70] C. P. Burgess, M. Cicoli, and F. Quevedo, "String inflation after Planck 2013," Journal of Cosmology and Astroparticle Physics, vol. 2013, no. 11, article 003, 2013. 
[71] J. P. Conlon and F. Quevedo, "Kähler moduli inflation," Journal of High Energy Physics, vol. 2006, no. 1, article 146, 2006.

[72] J. P. Conlon, R. Kallosh, A. Linde, and F. Quevedo, "Volume modulus inflation and the gravitino mass problem," Journal of Cosmology and Astroparticle Physics, vol. 2008, no. 9, article 011, 2008.

[73] E. Silverstein, "Simple de sitter solutions," Physical Review D, vol. 77, no. 10, Article ID 106006, 2008.

[74] E. Silverstein and A. Westphal, "Monodromy in the CMB: gravity waves and string inflation," Physical Review D, vol. 78, no. 10, Article ID 106003, 2008.

[75] W. Buchmuller, C. Wieck, and M. W. Winkler, "Supersymmetric moduli stabilization and high-scale inflation," Physics Letters B, vol. 736, pp. 237-240, 2014.

[76] W. Buchmüller, E. Dudas, L. Heurtier, and C. Wieck, "Largefield inflation and supersymmetry breaking," Journal of High Energy Physics, vol. 2014, no. 9, article 053, 2014.

[77] A. Hebecker, P. Mangat, F. Rompineve, and L. T. Witkowski, "Tuning and backreaction in F-term axion monodromy inflation," Nuclear Physics B, vol. 894, pp. 456-495, 2015.

[78] W. Buchmüller, E. Dudas, L. Heurtier, A. Westphal, C. Wieck, and M. W. Winkler, "Challenges for large-field inflation and moduli stabilization," Journal of High Energy Physics, vol. 2015, no. 4, article 058, 2015.

[79] E. Dudas and C. Wieck, "Moduli backreaction and supersymmetry breaking in string-inspired inflation models," Journal of High Energy Physics, vol. 2015, no. 10, article 062, 2015.

[80] G. R. Dvali, Q. Shafi, and R. K. Schaefer, "Large scale structure and supersymmetric inflation without fine tuning," Physical Review Letters, vol. 73, no. 14, pp. 1886-1889, 1994.

[81] M. Bastero-Gil, S. F. King, and Q. Shafi, "Supersymmetric hybrid inflation with non-minimal Kähler potential," Physics Letters B, vol. 651, no. 5-6, pp. 345-351, 2007.

[82] M. Kawasaki, M. Yamaguchi, and T. Yanagida, "Natural chaotic inflation in supergravity," Physical Review Letters, vol. 85, no. 17, pp. 3572-3575, 2000.

[83] M. Yamaguchi and J. Yokoyama, "New inflation in supergravity with a chaotic initial condition," Physical Review D, vol. 63, Article ID 043506, 2001.

[84] P. Brax and J. Martin, "Shift symmetry and inflation in supergravity," Physical Review D, vol. 72, no. 2, Article ID 023518, 24 pages, 2005.

[85] R. Kallosh and A. Linde, "New models of chaotic inflation in supergravity," Journal of Cosmology and Astroparticle Physics, vol. 2010, no. 11, article 011, 2010.

[86] R. Kallosh, A. Linde, and T. Rube, "General inflaton potentials in supergravity," Physical Review D, vol. 83, no. 4, Article ID 043507, 2011.

[87] E. Dudas, “Three-form multiplet and inflation," Journal of High Energy Physics, vol. 2014, no. 12, article 014, 2014.

[88] L. Aparicio, F. Quevedo, and R. Valandro, "Moduli stabilisation withnilpotent goldstino: vacuum structure and SUSY breaking," http://arxiv.org/abs/1511.08105. 

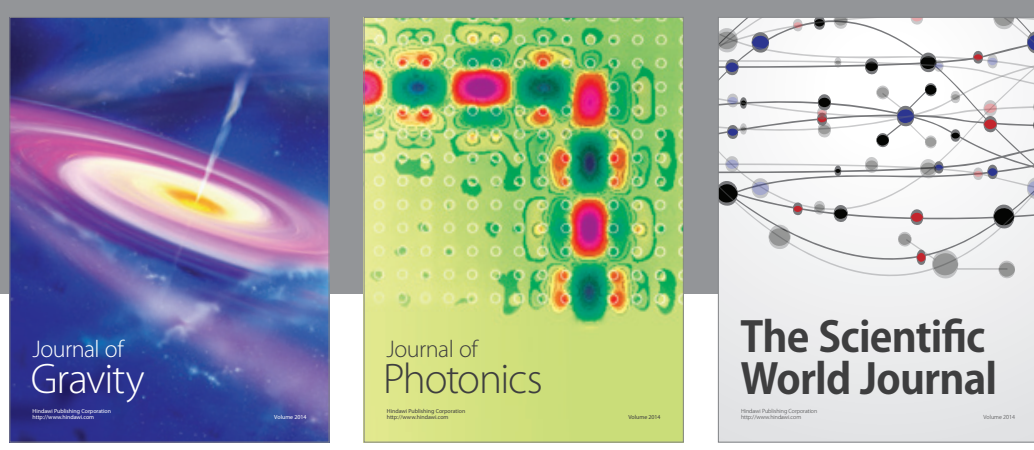

The Scientific World Journal
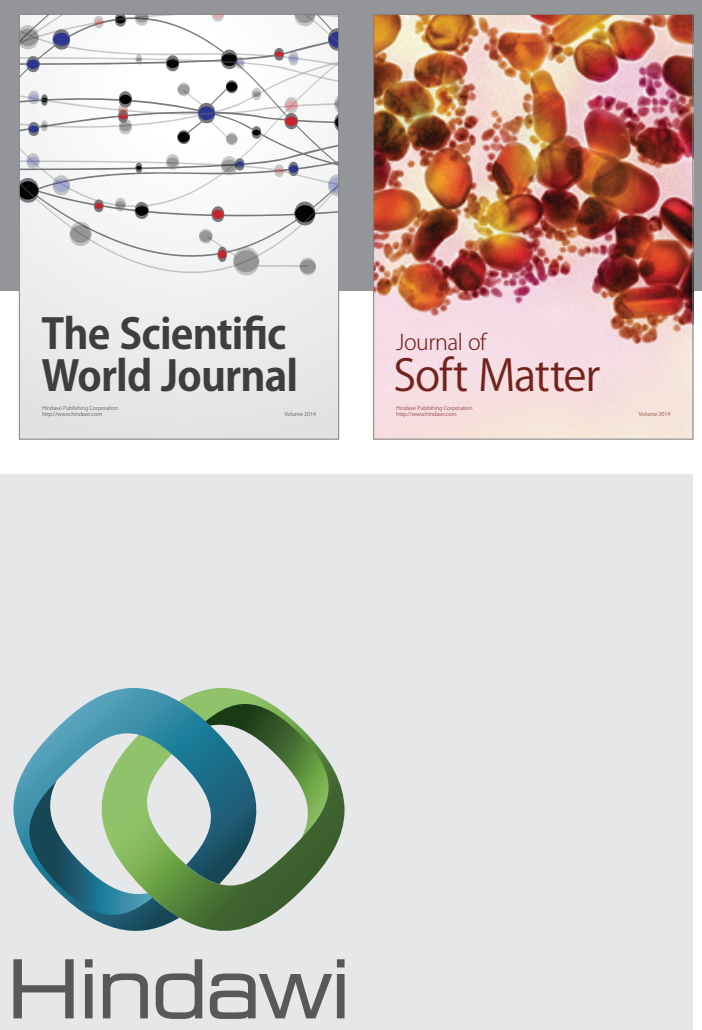

Submit your manuscripts at

http://www.hindawi.com

nternational Journal of

Statistical Mechanics
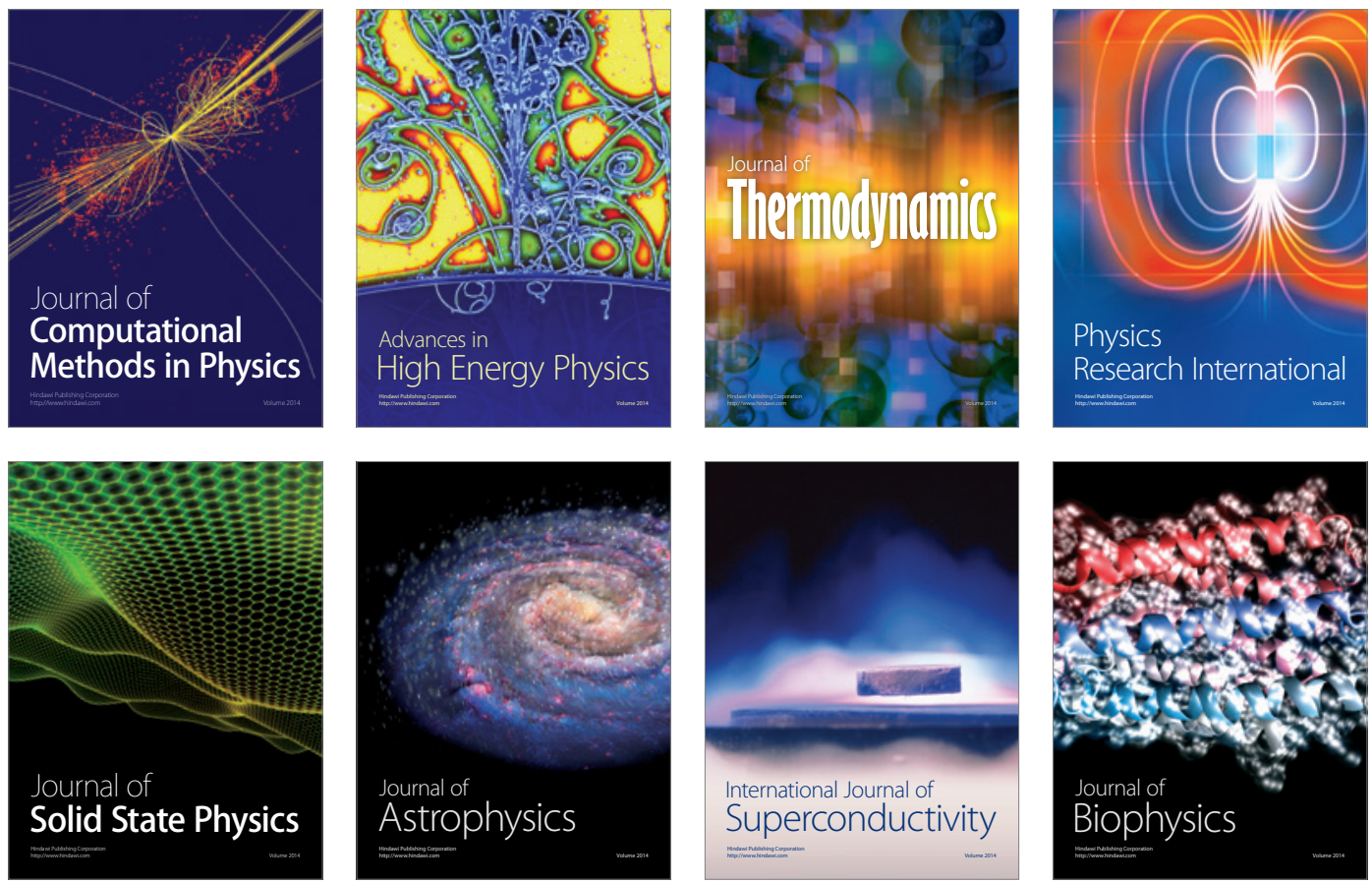
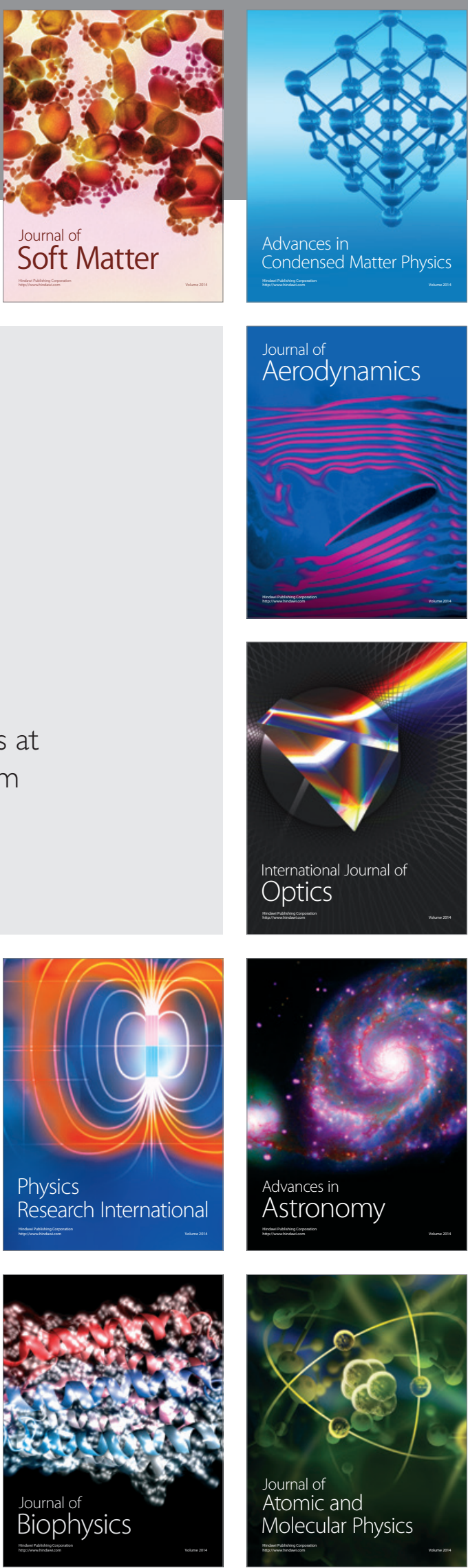